Article

\title{
Examining Evacuee Response to Emergency Communications with Agent-Based Simulations
}

\author{
C. Natalie van der Wal ${ }^{1, *(\mathbb{D})}$, Daniel Formolo ${ }^{2} \mathbb{D}$, Mark A. Robinson ${ }^{3}$ and Steven Gwynne ${ }^{4,5}$ \\ 1 Multi-Actor Systems Department, Faculty of Policy, Management and Technology, \\ Delft University of Technology, 2628 BX Delft, The Netherlands \\ 2 Department of Computer Science, Faculty of Science, Vrije Universiteit Amsterdam, \\ 1081 HV Amsterdam, The Netherlands; formolo@gmail.com \\ 3 Socio-Technical Centre, Leeds University Business School, University of Leeds, Leeds LS6 1AN, UK; \\ m.robinson@lubs.leeds.ac.uk \\ 4 Mövement Strategies, London EC1N 8TE, UK; steve.gwynne@ghd.com \\ 5 Division of Fire Safety Engineering, University of Lund, Box 117, 22100 Lund, Sweden \\ * Correspondence: c.n.vanderwal@tudelft.nl
}

check for

updates

Citation: van der Wal, C.N.; Formolo, D.; Robinson, M.A.; Gwynne, S. Examining Evacuee Response to Emergency Communications with Agent-Based Simulations.

Sustainability 2021, 13, 4623.

https://doi.org/10.3390/su13094623

Received: 9 March 2021

Accepted: 9 April 2021

Published: 21 April 2021

Publisher's Note: MDPI stays neutral with regard to jurisdictional claims in published maps and institutional affiliations.

Copyright: (C) 2021 by the authors. Licensee MDPI, Basel, Switzerland. This article is an open access article distributed under the terms and conditions of the Creative Commons Attribution (CC BY) license (https:// creativecommons.org/licenses/by/ $4.0 /)$.

\begin{abstract}
To improve communication during emergencies, this research introduces an agent-based modeling (ABM) method to test the effect of psychological emergency communication strategies on evacuation performance. We follow a generative social science approach in which agent-based simulations allow for testing different candidate solutions. Unlike traditional methods, such as laboratory experiments and field observations, ABM simulation allows high-risk and infrequent scenarios to be empirically examined before applying the lessons in the real world. This is essential, as emergency communication with diverse crowds can be challenging due to language barriers, conflicting social identities, different cultural mindsets, and crowd demographics. Improving emergency communication could therefore improve evacuations, reduce injuries, and ultimately save lives. We demonstrate this ABM method by determining the effectiveness of three communication strategies for different crowd compositions in transport terminals: (1) dynamic emergency exit floor lighting directing people to exits, (2) staff guiding people to exits with verbal and physical instructions, and (3) public announcements in English. The simulation results indicated that dynamic emergency exit floor lighting and staff guiding people to exits were only beneficial for high-density crowds and those unfamiliar with the environment. Furthermore, English public announcements actually slowed the evacuation for mainly English-speaking crowds, due to simultaneous egress causing congestion at exits, but improved evacuation speed in multicultural, multilingual crowds. Based on these results, we make recommendations about which communication strategies to apply in the real world to demonstrate the utility of this ABM simulation approach for risk assessment practice.
\end{abstract}

Keywords: agent-based model; crowd management; crowd simulation; emergency communication

\section{Introduction}

Ineffective emergency evacuations can lead to casualties, particularly when people delay escaping or remain in dangerous situations. Effective emergency preparedness is therefore important to help people perceive dangerous incidents more accurately and guide their evacuation in accordance with procedures. Existing emergency communication and crowd management guidelines indicate that messages should be clear, reliable, and prompt [1-6]. However, these guidelines rarely address cultural aspects, such as language barriers, cultural background, nationality, and social and demographic factors [7] that can influence the understanding of, and compliance with, evacuation instructions [8-12]. In this research, we focus specifically on cultural factors and language differences.

Human experiments to test the effectiveness of evacuation communication can be costly and ethically problematic as they may distress participants. Other approaches to 
examining emergency communications and evacuation behavior include interviews and surveys with emergency survivors and experts to create formal risk analysis models [13-17]. However, simply reviewing best practice and real-life failures in this way does not yield sufficient data to test emergency communication effectiveness systematically. Moreover, interviews with survivors of emergencies can also be unreliable as they depend on memory and accurate recall, which is known to be flawed especially after trauma $[18,19]$. Analysis of real-world emergency videos is one promising approach with high ecological validity [20], but this is still correlational rather than experimental.

Evacuation researchers have also used egress drills to collect data, and these have been a convenient laboratory. However, given the nature of drills, they typically limit the control that researchers can maintain over the conditions present (preventing true experiments), limit the types of scenario that can be examined (given ethical concerns), and limit the number of repetitions (given financial and fatigue concerns). Virtual reality (VR) and "serious games" are now being used to combat these concerns, providing a much more controlled, inexpensive environment [21]. However, there are still concerns about the representativeness of the conditions faced and of the decisions made in such conditions [22].

Consequently, to address these methodological limitations, we show here how agentbased modeling can be used to test various theoretically derived hypotheses regarding evacuee response using simulations of different scenarios. While such theories were often originally derived from conventional experiments, it is necessary to go beyond this original method to examine the array of scenarios necessary to test the robustness of the theory credibly and comprehensively. We show how theories can be embedded within ABM-of sufficient scope, refinement, and representativeness-to examine how reflective they are of real-world conditions. Our key concern here is to examine and differentiate between different theories, rather than definitively state the impact of a specific theory, however, to identify good candidate solutions for further analysis.

ABM is a scientific methodology that can explain observed meta-level phenomena from the behavior of micro-level actors [23,24]. The aggregate level patterns are called emergent effects that cannot be predicted directly from the individual behaviors or action selections, but rather emerge through interaction between individual actors [25]. ABM explains these emergent effects and also tests hypotheses about how they emerged. It integrates both quantitative and qualitative information from different disciplines and can distribute these stochastically to cope with ambiguities in our understanding and changes in the initial conditions.

ABM experiments are run in silico to approximate real life and can easily be repeated multiple times to collect more data or test different experimental conditions. Although the results are estimations, this article attempts to assess the credibility of the model by (1) including credible real-world factors, (2) representing their impact at an individual level (albeit in a simplified manner based on current theory and empirical evidence), and (3) comparing the aggregate outcomes with real-world expectations. Of course, by definition, such testing is partial and provisional, requiring continued testing efforts enabling more refined insights, increased confidence through repetition, and expanding the scenarios examined (e.g., [26]). As with all models, further real-world data are necessary for a more thorough validation. We employ ABM here to simulate emergent effects arising from different emergency communication strategies in various simulation scenarios [24].

Using ABM is still relatively novel in behavioral science, partly due to the interdisciplinary expertise needed for such an approach [27]. As a non-traditional approach to theory development, it must overcome skepticism. Both qualitative and quantitative information from different disciplines—such as concepts like social identity, languages, and risk probabilities - need to be integrated into one computational and temporal model to dynamically predict outcomes for different crowd compositions.

Here, we try to simulate the effectiveness of emergency communications through agent-based simulations. Although ABM has previously been used to study emergency 
communication response [28-30] and risk analysis [31], this ABM work is particularly novel as it examines socio-cultural aspects in emergency communication with multi-cultural crowds, which would not be practical to explore in sufficient detail and scope with traditional methods. This approach also allows us to specify and refine which (expensive) human experiments to conduct in future, with each method feeding on and off the other in an iterative and integrated manner [25].

Based on the most feasible proposed strategies for large transport terminals from a previous literature review [32], we chose to test the effectiveness of the following three communication strategies in a simulated environment: (1) dynamic emergency exit floor lighting directing people to the exit, (2) staff located at exits giving verbal and physical evacuation instructions, and (3) a clear spoken English public announcement to evacuate calmly to the nearest exit. For each communication strategy, our research question is: what is the effect of the emergency communications on the total evacuation time and number of falls? Total evacuation time was chosen to compare our model's outcomes with a benchmark from previous work [33] and measured as the time from the onset of danger until the moment all agents have evacuated the building. Number of falls was chosen as it was of interest to our stakeholders so they could relate falls to age and groups. Total number of falls was measured as the sum of all falls in a single simulation run, each fall for each agent counting toward the total number of falls. Other metrics could also have been examined. We tested this with structured agent-based simulations for different crowd compositions, as outlined next in the Method section. These simulations would present a challenging and expensive set of experimental tests using human participants but become more viable and cost-efficient when using the approach employed here.

\subsection{Solutions for Emergency Communications}

We are specifically investigating emergency communication in large buildings where multicultural crowds gather and have therefore selected international transport terminals as our context here. Fridolf et al. [34] indicated that passengers usually wait for staff instructions before evacuating (a role-rule model); usually form groups, evacuate using familiar exits, and prefer remaining in familiar places (an affiliative model); evacuate more if others do so (an informational social influence model); and seek environmental information to interpret the situation, to decide on and initiate actions (a behavior sequence model).

Complementing these observations, in [34-36] it is claimed that inappropriate staff responses could even induce long evacuation delays while positive staff actions facilitate the evacuation. These potentially risky behaviors during evacuation could be prevented with emergency communication solutions for multicultural crowds. For example, emergency floor lighting directed at the exit can compensate for language issues that might undermine the effectiveness of text instructions. [37,38]. Typically, such lighting uses light emitting diodes (LED) in lines on the floor that strobe dynamically to trace a direct path to the appropriate emergency exit (e.g., as examined in [39]).

\subsection{Socio-Cultural Factors}

Cultural factors in crowd management should be considered to prepare effectively for different risk scenarios [40]. For example, due to various backgrounds, language proficiencies, and educational levels, people might not understand verbal emergency instructions or signs and therefore not respond appropriately [41].

Another example is that people might be used to staying together in groups or be unfamiliar with the environment, such as when family groups or tourists travel or visit an event [42]. In emergencies, it is important that people understand instructions, can make individual decisions, and do not block the flow of other people evacuating [43]. It is also important, for example, to prepare for families or other groups waiting for, or collecting, their members during evacuations or moving at their own pace, instead of assuming everybody will immediately evacuate (e.g., [44]). 
Additionally, research suggests that cultural factors, such as social identity, can play an important role not only in the way people behave within crowds [45], but also in emergency evacuation situations [46] due to factors such as different risk attitudes linked to gender or culture [47]. Comprehension of signs can also vary due to age, literacy, or education [48,49].

Five key socio-cultural issues from the research literature relevant for emergency communication with crowds are shown in Table 1 [50]. We define culture here as "the collective programming of the mind that distinguishes the members of one group or category of people from another" [50]. While identity can correspond to nationality, Hofstede [51] believes this definition also applies to collectives such as regions, ethnicities, occupations, organizations, or even ages and genders.

Table 1. Overview of key socio-cultural issues in crowd management, and associated recommendations for emergency communication, table reproduced with permission of the authors [50].

\begin{tabular}{|c|c|}
\hline Category & Summary of Cultural Factors and Recommendations \\
\hline Social identity & $\begin{array}{l}\text { Encouraging a united crowd identity will stimulate more cooperation and helping of fellow in-group } \\
\text { members [21]. } \\
\text { Crowd management staff should engage with crowd members to get them on their side and encourage } \\
\text { cooperation (e.g., [44,52]). }\end{array}$ \\
\hline Language & $\begin{array}{l}\text { Communication with passengers should use simple and widely understood languages to: (1) decrease } \\
\text { response time, (2) reduce crowd density, and (3) discourage the use of familiar yet dangerous routes. }\end{array}$ \\
\hline Signage & $\begin{array}{l}\text { Messages and instructions on signs should be universally understood, using clear symbols where possible } \\
\text { (e.g., [53]). }\end{array}$ \\
\hline Landmarks & Clear landmarks should be used to guide multi-cultural passengers in unfamiliar environments (e.g., [54]). \\
\hline Communication & $\begin{array}{l}\text { Message content and crowd management procedures should be adapted to crowd demographics. } \\
\text { Communication should be tailored to different subgroups, adjusting content and tone [55]. }\end{array}$ \\
\hline
\end{tabular}

\subsection{Related Work}

There are many different approaches for modeling building evacuations, such as microscopic models (e.g., cellular automata, social force, agent-based models), or macroscopic models (e.g., fluid dynamics) [56]. Templeton et al. [57] reviewed crowd models and concluded that current crowd simulations do not include psychological factors and therefore cannot accurately simulate the collective behavior that has been found in extensive empirical research on crowd events. Kuligowski [58] reviewed 28 egress models and categorized them into behavior or movement models, where either evacuee decisionmaking was modeled or where the focus was on movement out of a building. In [7], social and psychological factors incorporated into evacuation simulation models were reviewed. Some models (e.g., FIRESCAP, EXODUS, MASCM) simulate group decision making, while other models (e.g., EGRESS, SIMULEX, EXIT89) focus more on physical constraints and factors such as walking speed and stairways to find the optimal flow in the evacuation process. Even when evacuation models have parameters such as gender, age, and individual walking speeds, they can lack socially interactive characteristics such as monitoring others, directing, collective evaluation, and collective agreement on appropriate responses. According to [7], from a social science perspective, the ideal simulation modeling approach for realistic crowd models should seek the development of sub-models that posit an active, investigative, socially-embedded agent that assesses the state of other agents (people) and forms a definition of the situation in cooperation with others. It would recognize that group dynamics is an essential dimension that must be considered, such as in emergent norm theory. Addressing these two recommendations [7], it was decided to use the IMPACT model in this project [33], as it models group decision making, including emotional and informational social influence. Other evacuation models do not include collective agreement based on emotions and information in the evacuee decision-making process. 


\section{Materials and Methods}

Based on reviews of crowd simulations, to enable important processes to be represented and emergent properties to be captured, a model should include psychological factors and social factors (enabling group dynamics), and allow for agents to make decisions based on the existence and actions of those around them [7,56-58]. Accordingly, we selected the agent-based IMPACT model for our evacuation simulations, which incorporates these social dimensions [33]. As part of the validation process, the IMPACT model was compared against a credible physical benchmark-the application of the EXODUS evacuation model — of a real-world evacuation on a ship with multiple decks and different escape routes $[59,60]$. The IMPACT model covers more social dimensions than the benchmark EXODUS model (e.g., group decision-making, social, and informational interactions); therefore, different versions of the IMPACT model—with and without social influence-were compared to the benchmark, to enable a fair comparison-especially of its capacity to reasonably represent physical conditions. For the IMPACT model without social influence, a selection of the model's variables, such as response time and speed, were fixed to the benchmark settings, while in the "IMPACT model with social influence" condition, these were calculated according to the IMPACT model itself.

Results of these comparisons showed that both versions of the IMPACT model一with and without social influence-were capable of accurately simulating the assembly curves and total evacuation times compared to the EXODUS model, as they stayed within the approved boundaries given by the validation dataset [60]. Moreover, the IMPACT model results showed that for this ship evacuation, the passenger agents evacuated faster when social influences were modeled versus when they were not, which was explained as a result of the group decision making processes influencing response times and route choices. Other what-if scenarios were simulated with the IMPACT model, and sensitivity analyses showed that certain social dimensions-namely social spread of emotions and information, travelling in groups, and familiarity with the environment-can have a significant effect on the model outcomes, reducing evacuation time by up to $30 \%$. The specific impact will vary according to the scenario conditions. These results show the importance of including social dimensions in evacuation simulation models.

The design of the IMPACT model was informed by the agent-based belief-desireintention (BDI) and network-oriented modeling approaches [61,62]. Here, group decision making is modeled as the social influence of cognitive and emotional states, and the effect of beliefs and emotions on individual decision making (e.g., influenced by the identity of surrounding agents). The model incorporates the following social dimensions: familiarity, response time, helping behavior, culture, falls, gender, and age. Familiarity represents how familiar an agent is with the environment, which influences the exit choice. Response time represents the pre-evacuation delay, starting from observing the danger or hearing the alarm until starting to move toward an exit. Helping behavior was modeled as passenger agents assisting another fallen passenger agent to stand up (given that they had fallen). Culture was modeled as each agent belonging to a cultural cluster, which influenced the English proficiency of the agent, which in turn influenced their understanding of English public announcements. Falls, gender, and age were modeled as the ability of agents to stand back up after falling, which was influenced by their age and gender. In this instance, these are assumptions that might be modified according to new insights, information, and scenario conditions.

\subsection{Brief Overview of ABM Implementation of Emergency Communications and Evacuee Response}

In previous research [54], the IMPACT model simulated crowd members evacuating from an empty room without environmental effects, and examined the effects of each social and cultural parameter (independent variables) on the evacuation time (dependent variable). This previous, first version of the IMPACT evacuation model is the domain 
model, which has been validated against real world data [33] and was implemented in the NetLogo programming language, version 5.3.3.

Our current research builds upon this earlier work, using the domain model as our methodological platform, extending it by now incorporating environmental effects (e.g., different emergency communications) on the behavior of the agents. The domain IMPACT model has been extended with new rules and states that make it possible for agents to react to new environmental communication features (public announcements, emergency lighting, and staff members at exits). This represents a methodological development of the original model, that is then used to test the hypotheses reported here.

When running simulations with the IMPACT evacuation model, the user can specify the following settings for the characteristics of the agents (representing people) during an evacuation simulation: (1) age group (children, adults, or elderly); (2) gender (male or female); (3) compliance level (modeled as a number between 0 and 1, depending on age and gender, based on $[63,64]$ and having an amplifying effect on the desire to evacuate); (4) cultural group (modeled as belonging to one of the 11 cultural clusters [60]); (5) number of groups, comprising 2,3, or 4 members; (6) number of people who do not belong to a group; (7) environmental familiarity (on or off); (8) falls (on or off); and (9) social influence, which represents the spread of emotions and information through the crowd (on or off). The settings of the current experiments are shown in Tables A1 and A2.

The IMPACT evacuation model can generate a set of outputs, which are operationalized as follows.

Individual evacuation time: Elapsed time (ticks) from the onset of danger until the moment the living agent evacuates the room.

Total evacuation time: Elapsed time (ticks) from the onset of danger until the moment the last living agent evacuates the room.

Individual response time: Represents pre-evacuation delay, which can be measured as the time from the start of the danger or the perception of danger until the start of movement towards an exit. For each agent, this can be measured as starting at the start of the danger, when first observing the danger, when first being informed of the danger by another agent, or when perceiving the alarm, and ending at the moment the agent starts moving toward an exit.

Average response time: The average of all individual response times.

Total number of falls: The total number of falls of all agents. Each fall is counted.

Number of evacuees per exit: Number of evacuated agents per exit, plotted over time for each time step of the simulation run.

Egress rate through each exit: Number of agents leaving via a particular exit, plotted over time for each time step of the simulation run.

Average speed of individual agents: The average walking speed of an agent, plotted over time during each time step of the simulation run.

Individual fear level: The level of fear (operationalized in the model as a number within the range 0 to 1 ), plotted over time for each time step of the simulation run.

Average intention to evacuate. The level of intention to evacuate (operationalized in the model as a number within the range 0 to 1 ), plotted over time for each time step of the simulation run.

The configurable environmental characteristics for our current simulations are as follows: (1) population (total number of people); (2) number of staff members per exit $(0,1,2,3$, or 4$)$; (3) incident location (any location in the grid, a minimum of $4 \mathrm{~m}$ away from an exit, to allow agents to reach the exit); (4) number of exits (2 or 4); (5) type of environment (square or rectangular room, or own imported room shape); (6) presence of alarm (present or absent); (7) start time of alarm; (8) lighting guidance (on or off); and (9) public announcements (on or off). Some of these features are set on the graphical interface (see Figure 1), while others are configured in a config file. The IMPACT model can be run in different environments, such as for different buildings and with multiple floors, as was done in the validation study with a ship environment with multiple decks. In these 
experiments, we chose single rooms with multiple exits. In this way, we were able to determine the pure effects of different communications on evacuation behaviors, without interaction effects of the environment, such as stairs, hallways, and multiple floors.

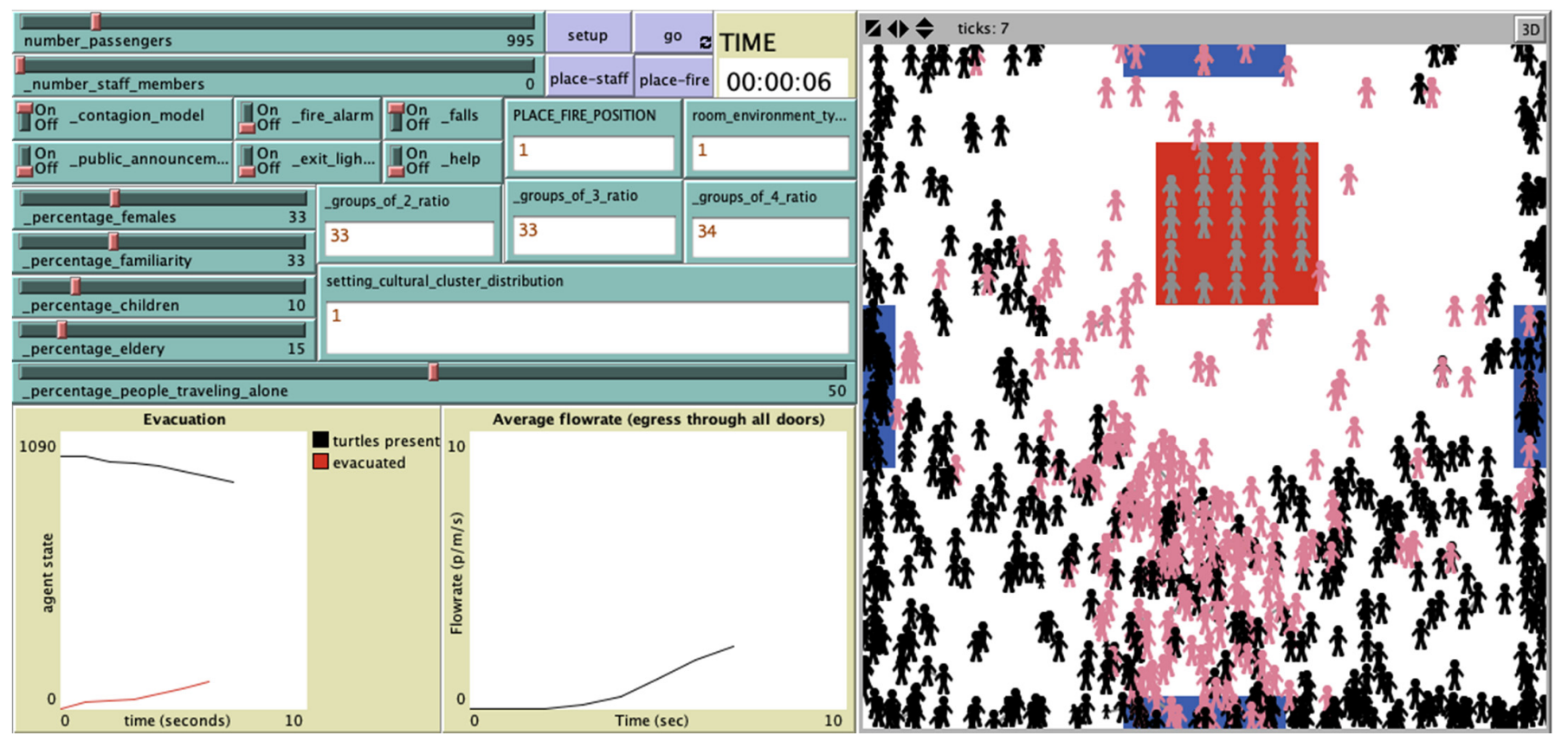

Figure 1. Screenshot of evacuation simulation in the IMPACT model: black agents are walking randomly, pink agents have started to evacuate, grey agents are deceased, the red square represents a danger, blue rectangles represent exits.

In summary, the observations of other agents (information and emotion), signs of danger (information from others, alarm, the danger itself), and internal states (levels of fear, beliefs about how dangerous the situation is, and intention to evacuate) together lead to the agent moving toward an exit, or not.

Figure 2 shows the activity diagram of the simulation, focusing on the internal model, system updates, internal states, and actions of each agent. The simulation starts with setting up the environment and agents, then each agent observes its environment, calculates its internal states and is able to fall or help a fallen agent. Next, the agent decides between moving randomly or evacuating; the system controls the egress at the exits so that only a certain number of agents can evacuate per time step and the environment is updated. At the same time, through the user interface, the environment can be updated as well through a feature of NetLogo, where a user can change settings through buttons or sliders, such as starting the incident or sounding the alarm, which will happen in real-time. Then statistics are updated, it is checked whether agents are dead or still alive and the cycle starts again. After the simulation has run, the model finalizes the cycle and updates the statistics. The simulation stops when all agents have either evacuated or are not able to evacuate. At any moment, the user can change the parameters available on the interface and influence the environment or agents.

For all of our experiments, we simulated an environment with a square layout $(20 \times 20 \mathrm{~m})$ with four exits (top, down, left, right; main exit $=$ down), representing a general building such as a transport terminal. The simulation scenario represented a fire in a transport terminal that is present from the first second (tick) in the simulation. After $3 \mathrm{~min}$, the evacuation alarm sounds in the simulated environment. The socio-cultural factors influencing the evacuation time are: (1) environmental familiarity, (2) social influence, (3) crowd density, (4) gender, (5) age, and (6) grouping behavior. The following three communication solutions were systematically introduced across the different experimental conditions: (1) dynamic emergency exit floor lighting directing people towards the emergency exits, 
(2) staff at exits guiding people out, and (3) a public evacuation announcement in English. We experimentally examined the effect of each of these three emergency communication strategies (independent variables) on the total evacuation time and number of falls (dependent variables). We provide more details of the parameter settings for the simulations in the next sub-section, but first we explain how we implemented the emergency communication solutions in our model.

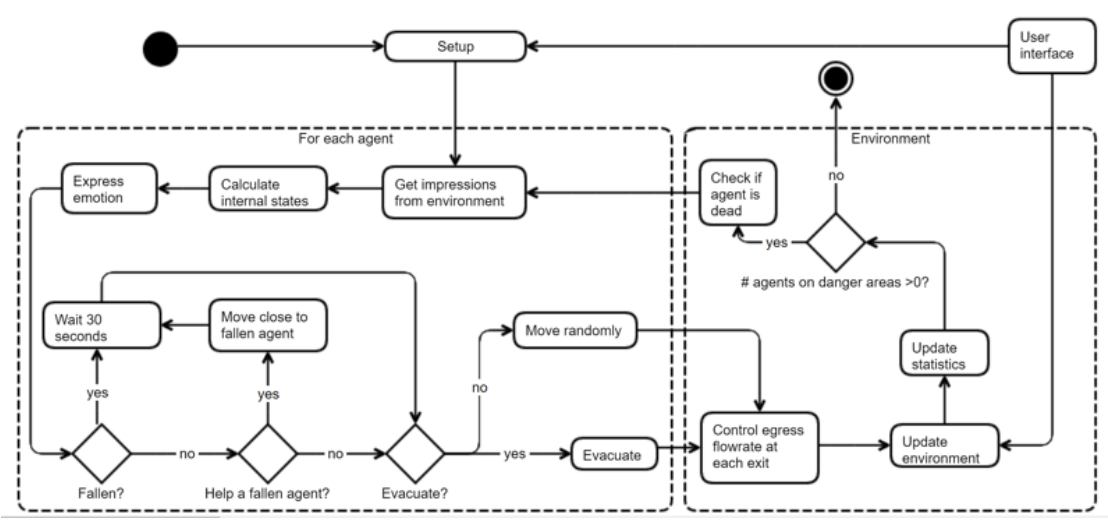

Figure 2. General activity diagram of the evacuation simulator, showing how the model setup and user interface settings influence the agent and environmental states.

ABM Implementation of New Emergency Communications

First, we extended the model from van der Wal et al. [33] with new concepts and relations. Figure 3 shows the original model in white and dark grey nodes and the extended part of the model in light grey nodes. The diagram shows the network-oriented modeling approach in which nodes are concepts and the arrows represent the relationships between them. All concepts are modeled as a number with range 0 to 1 , where 0 means the state is minimal and 1 means the state is maximal. For example, the state belief_dangerous represents the agent's belief about whether the situation is dangerous or not, and is calculated as a weighted combination of the agent's three observation states: (1) observation_alarm, (2) observation_fire, and (3) observation_others_belief_dangerous. For a more extensive and detailed explanation of the model's development, see [33] and its validation in [65]. The light grey and dark grey nodes and linking arrows represent the extended part of the model for the emergency communication solutions. Light grey nodes are new concepts (see Table 2), and dark grey nodes are existing concepts in the domain model (nationality, environmental familiarity, move to exit).

Next, we created IF-THEN rules to define relationships between concepts, a standard feature of ABM (e.g., [25]), needed for the hypotheses we wanted to test. These rules are listed in Table 3 in pseudo-code - an accessible verbal translation of the programming code. We implemented these rules in the model using the NetLogo programming language.

Dynamic emergency exit floor lighting. The first IF-THEN rule in Table 3 is for the dynamic emergency exit floor lighting guiding people to the exits. Whenever an agent sees these dynamic emergency exit floor lighting LED strips, the agent will take the nearest exit indicated by the lighting. This rule was based on studies determining that $38 \%$ of people see standard emergency signs while $77 \%$ of people see a dynamic emergency sign, and when people see either sign, 100\% follow it [66]. Research has shown that even in a virtual, smoky tunnel environment, $100 \%$ of participants noticed the continuous LED strips and they had a positive impact on evacuation time and walking patterns [67]. Accordingly, the agents in our simulated environment must be in close vicinity of the dynamic lighting to see it and then follow it to the exit. Close vicinity was modeled as the dynamic light strip being in the current grid cell (patch) that the agent is standing on, or in one of its eight neighboring grid cells, corresponding to a distance of $1 \mathrm{~m}$. An example of a simulation run including emergency lighting is shown in Figure 4. In the simulations, once an agent notices the lighting, it will follow the lights until the exit, even if it knows 
another exit, as it assumes that the lighting will guide it to the nearest exit. Note that the compliance with lighting instructions is different from the general compliance level in the model. The general compliance level of the passenger agents was modeled as a number between 0 and 1, having an amplifying effect on the desire to evacuate. The level depends on the agent's gender and age (e.g., males are less compliant than females, based on data from [63]), modified for different age groups using data from [64]. Therefore, this general compliance level already incorporates variation in whether agents decide to evacuate or not. Then, once an agent is evacuating, the agent is $100 \%$ compliant with following the emergency lighting if they have observed it, as based on empirical studies. As mentioned previously, these responses can be modified to reflect new data and understanding.

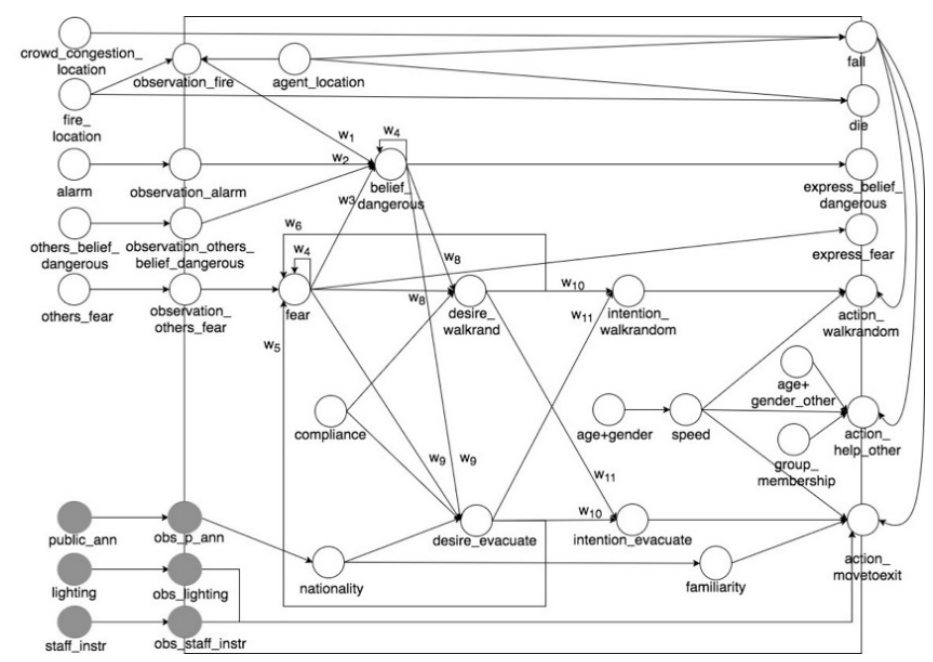

Figure 3. Evacuation Domain Model (white concepts indicate the evacuation process in a normal situation; [33]) and Support Model (grey concepts that are necessary for the emergency communication strategies). The labels of the concepts are the formal names used in the model. The arrows indicate how the concepts are related and influence each other.

Table 2. Implemented concepts necessary for the IF-THEN rules (Table 3).

\begin{tabular}{cccc}
\hline Type & Concept & Value & Meaning \\
\hline Event & public_ann & 0 or 1 & $0=$ no announcement; $1=$ clear public English announcement. \\
Event & lighting & 0 or 1 & $0=$ no lighting; $1=$ emergency lighting on the floor indicating exits. \\
Event & staff_instr & 0 or 1 & $0=$ no staff at exit; $1=$ staff at each exit instructing evacuation. \\
Perception & obs_p_ann & 0 or 1 & $0=$ not observed; $1=$ observed. \\
Perception & obs_lighting & 0 or 1 & $0=$ not observed; $1=$ observed. \\
Perception & obs_staff_instr & 0 or 1 & $0=$ not observed; $1=$ observed. \\
\hline
\end{tabular}

Table 3. IF-THEN rules implemented in the agent-based model (pseudo-code).

\begin{tabular}{cc}
\hline No. & Rule \\
\hline 1 & IF emergency lighting is on AND a passenger can see the emergency lighting \\
THEN he will follow it to the exit
\end{tabular}




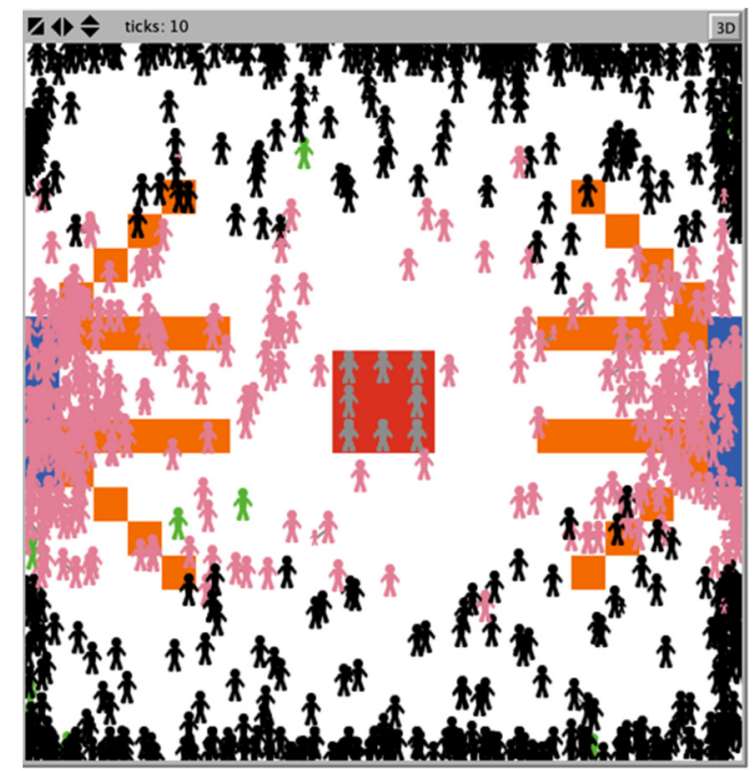

Figure 4. Simulation environment of the IMPACT model with two exits (blue patches), equipped with emergency lighting (orange patches).

Staff member guidance. The second IF-THEN rule in Table 3 is for the staff member agents present at the exit. The implementation is based on research by Shields and Boyce [36], indicating that staff members have a positive impact on evacuation time and compliance with instructions. Specifically, they found that, on average, $50 \%$ of evacuees received their first indication of an emergency from being told by staff, rather than hearing the alarm, seeing others moving towards the exits, or being told by other shoppers. Furthermore, the participants' exit choices were dependent on staff instructions (25\%), selecting the nearest $(50 \%)$ or most familiar exit $(20 \%)$, or by following others $(5 \%)$. We translated these results as the passenger agent seeing the staff member agent when in close vicinity and then always following their instructions. Close vicinity was modeled as within a radius of 5 grid cells (patches), which represents $5 \mathrm{~m}$. The staff member agent is always within 2 grid cells (patches) of an exit, which represents $2 \mathrm{~m}$.

Public announcements. Finally, the third IF-THEN rule is for the last emergency communication solution strategy: the public announcement. It represents a public announcement made in English that tells passengers to "please stay calm and evacuate through the nearest exit". The probability of an agent understanding the message depends on the average English proficiency of the cultural cluster the agent belongs to. For each cultural cluster from Ronen and Shenkar [68], the English proficiency was calculated based on the EF English Proficiency Index [69] that rates countries by the average level of English language skills. Once the agent understands the message, it complies with it. The announcement was made $20 \mathrm{~s}$ after the fire alarm sounded and then repeated every $60 \mathrm{~s}$.

\subsection{Simulation Experiments}

After developing our ABM, we then used it to perform a series of structured experiments to examine the effect of the emergency communication. Although we performed these experiments as simulations using our ABM rather than with human participants, we still refer to them as experiments as we are using the scientific method by systematically manipulating features of emergency communication (our independent variables), to examine their effect on types of evacuee response (our dependent variables), using the agents in the model to represent crowd members. As such, they are experiments using a simulation model rather than a human model (with real participants employed in an artificial scenario, such as a laboratory experiment or evacuation drill). Nevertheless, for clarity, we refer to our experiments as simulation experiments specifically here to avoid 
potential confusion among readers from social sciences whose experiments would almost always involve human participants.

\subsubsection{Research Questions and Expected Outcomes}

We ran simulation experiments to test the effectiveness of the three communication strategies (our independent variables). Our first research question is, what is the effect of dynamic emergency exit floor lighting guiding people towards exits on the evacuation time and number of falls? The expected outcomes, based on empirical research, are (1) that evacuation time will be faster with dynamic emergency exit floor lighting than without (because lighting helps people see/find exits faster); (2) environmental familiarity will decrease evacuation time, in comparison with environmental non-familiarity; and (3) there will be no difference in the number of falls whether emergency lighting is present or not, as we have no a priori indications of this.

Our second research question is, what is the effect of staff guiding people towards exits on the evacuation time and number of falls? The expected outcomes, based on empirical research, are (1) the more staff, the shorter the evacuation time will be; (2) this decrease in evacuation time could be linear or non-linear; and (3) there will be no difference in the number of falls between when staff are guiding people or not.

Our third research question is, what is the effect of an English public announcement on the evacuation time and number of falls? The expected outcomes, based on empirical research, are that (1) English public announcements will reduce evacuation time when people understand the announcement, because people will start evacuating sooner and choose the nearest exit; (2) no differences in the number of falls are expected between English announcements or not.

\subsubsection{Outcome Measurements}

There are two outcome measurements (our dependent variables): (1) evacuation time, measured as the elapsed time from the onset of the fire until the last agent evacuates the room; and (2) total number of falls, measured as all falls in a single simulation run. Falling was implemented as follows. If an agent accelerates beyond its typical walking speed and reaches faster than $3 \mathrm{~m}$ per second $(\mathrm{m} / \mathrm{s})$, and there are more than four agents in the same square meter, then there is a $5 \%$ chance of falling for each new movement. Otherwise, the agent does not fall. This is an assumption and can be modified to examine the sensitivity of the number of falls generated to this parameter in conjunction with the other conditions present. When an agent falls, it takes $30 \mathrm{~s}$ before it stands up and moves again. We could not find any empirical data on the exact time it takes; therefore, we performed a pilot study ourselves that showed an average of $30 \mathrm{~s}$ to stand up. If another agent is helping, this is implemented as the agent waiting next to the fallen agent until it stands up. This represents the helper in real life assisting the fallen agent to stand up. Currently, it is possible for multiple agents to help a fallen agent at the same time, but due to the low probability it rarely happens in simulation runs.

As stated in the introduction, these outcome measurements were chosen to compare our model's outcomes with a benchmark from our previous work [33], and as it was of interest to our stakeholders. In addition, by choosing only two outcome measurements, it allowed us to manage multiple analyses and make comparisons across various parameters influencing these outcomes. We did consider other outcome measurements, however, including speed and the number of people reaching a safe zone, but they are less suitable for measuring our observed parameters' effects. For instance, defining a safe zone is not simple, as once it becomes congested, it could become hazardous (e.g., crushing) or even become a potential terrorist target. Furthermore, speed is also influenced by environmental features not present in our scenarios, such as barriers, walls, or flows in corridors. 


\subsubsection{Parameter Settings}

In each simulation experiment, certain factors are systematically varied (our independent variables), to examine their impact on the outcome measurements (our dependent variables). All experiments vary crowd density from low to medium to high, representing 2,4 , or 6 people per square meter, resulting in 800,1600, or 2400 people in total, respectively, at the beginning of the simulation. All other factors and levels are shown in the Appendix A, in Table A1. The demographics are set to reflect the standard European population composition, namely 15\% children, $15 \%$ elderly, and 70\% adults [70]. In Experiments 1 and 2, the population is divided evenly across all 11 cultural clusters [68]. In Experiment 3 , the crowd composition is varied by four different cultural cluster settings: $1=$ all passengers from the Anglo cluster (95.39\% English Proficiency); 2 = all passengers from the Eastern Europe cluster (16.28\% English Proficiency); $3=50 \%$ of the passengers from the Anglo cluster and 50\% from the Eastern Europe cluster; and $4=$ an even distribution between the 11 clusters [68].

The agent environment for the simulations was a square $\left(20 \times 20 \mathrm{~m}^{2}\right)$ layout of a building with four exits (top, down, left, right; main exit = down), representing a generic transport terminal. Environmental features such as stairs, slopes, corners, and counterflow passages were not included, to allow a more pure measurement of human behavior. Factors such as door width, gender, age, and compliance level were kept constant across all simulations (see Appendix A, Table A2).

The IMPACT model is a non-deterministic model. Observations and actions of a single agent (e.g., detecting the danger or not, finding the exit), and interactions between agents (e.g., through the spread of information, or emotion, and traveling in groups), lead to variations in the outcomes of simulation runs for the same scenario. To determine the number of simulation repetitions for each condition, we selected an evacuation scenario that we had found to generate maximum variability in the evacuation time output. Specifically, we used the default settings from the simulation experiments, for the largest environment, with the most exits, and largest number of passengers. We then ran this scenario 100 times to determine the exact number of repetitions required. First, we inspected the cumulative averages and variances in evacuation time to detect the number of repetitions at which evacuation time stabilized (which we verified). Second, we used Equation (1) to find the minimum number of repetitions (56) to guarantee that the error in the outcome result of one simulation run was within $5 \%$ of the maximum error of all runs with a $95 \%$ confidence level. Therefore, we chose 60 simulation repetitions for each condition, and then averaged the outcome results. We checked all simulations and they indeed stabilized after 60 repetitions.

$$
n \geq[100 \cdot Z \cdot S / r \cdot \bar{x}]^{2}=56.61599 \rightarrow 60 \text { samples }
$$

whereby, $Z=$ confidence interval of $95 \% ; s=$ standard deviation $=53.4287 ; r=$ maximum error of $5 \% ; \bar{x}=$ evacuation time average of 100 samples.

\section{Results}

For each of the three evacuation communication strategies, we now show the results addressing the research question, what is the effect of the emergency communication on the evacuation time and total number of falls?

\subsection{Simulation Experiment 1: Effectiveness of Dynamic Emergency Exit Floor Lighting}

First, a $3 \times 2$ independent ANOVA was performed on evacuation time, with crowd density (low, medium, and high), and emergency lighting (on, off) as between factors. The main effect and interaction effects were significant (see Table 4). Post hoc tests with Tukey HSD corrections for multiple comparisons show that, between conditions, the high level of crowd density differed significantly from low and medium: low-medium, $p=0.517$; medium-high, $p<0.001$; low-high, $p<0.001$. 
Table 4. Results simulation experiment 1: effectiveness of dynamic emergency exit floor lighting.

\begin{tabular}{|c|c|c|c|}
\hline Factor & $d f$ & $F$ & $p$ \\
\hline \multicolumn{4}{|c|}{ Effects of crowd density and emergency lightin on evacuation time, $3 \times 2$ ANOVA } \\
\hline Crowd density & 2 & 24.30 & 0.000 \\
\hline Emergency lighting & 1 & 6.73 & 0.010 \\
\hline Crowd density $\times$ Emergency lighting & 2 & 4.40 & 0.013 \\
\hline \multicolumn{4}{|c|}{ Effects of environmental familiarity and emergency lightin on evacuation time, $2 \times 2$ ANOVA } \\
\hline Environmental familiarity & 1 & 140.98 & 0.000 \\
\hline Emergency lighting & 1 & 7.54 & 0.006 \\
\hline Environmental familiarity $\times$ Emergency lighting & 2 & 8.94 & 0.003 \\
\hline \multicolumn{4}{|c|}{ Effects of crowd density and emergency lighting on evacuation time, $3 \times 2$ ANOVA } \\
\hline Crowd density & 2 & 330.49 & 0.000 \\
\hline Emergency lighting & 1 & 78.99 & 0.000 \\
\hline Crowd density $\times$ Emergency lighting & 2 & 41.81 & 0.000 \\
\hline \multicolumn{4}{|c|}{ Efefcts of environmental familiarity and emergency lighting on total number of falls, $2 \times 2$ ANOVA } \\
\hline Environmental familiarity & 1 & 146.06 & 0.000 \\
\hline Emergency lighting & 1 & 49.80 & 0.000 \\
\hline Environmental familiarity $\times$ Emergency lighting & 2 & 57.48 & 0.000 \\
\hline
\end{tabular}

These statistically significant results in Table 4 show that the emergency lighting (short for dynamic emergency exit floor lighting) decreased evacuation time in both medium and high crowd densities (see Figure 5). In high crowd density, crowd congestion appeared around the exits, but was relieved by the emergency lighting. The passengers followed the emergency lighting, which led to less variation in walking patterns and fewer falls. In the low crowd density condition, however, there was little crowd congestion, so the emergency lighting did not have an effect.

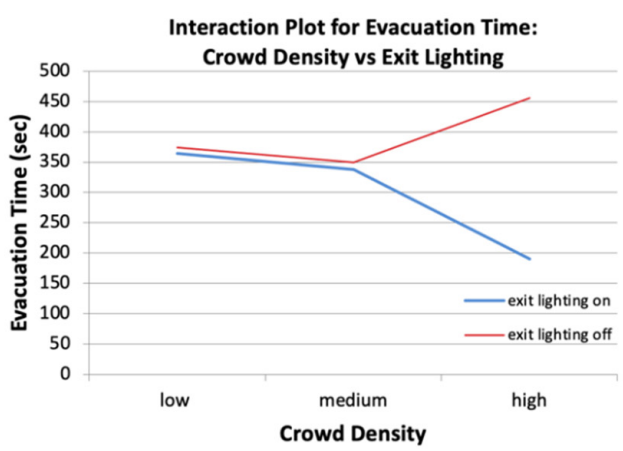

(a)

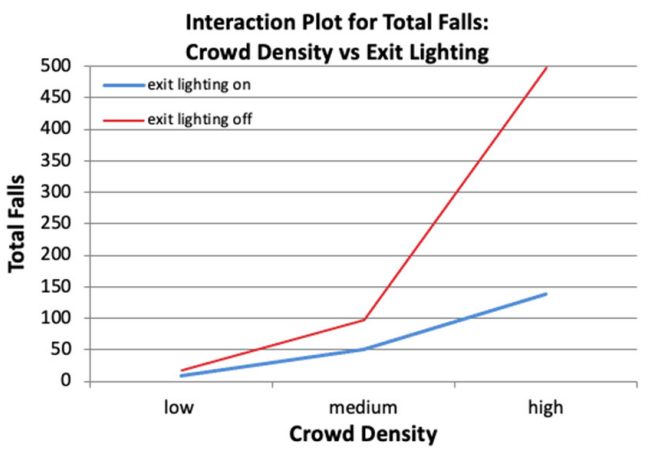

(c)

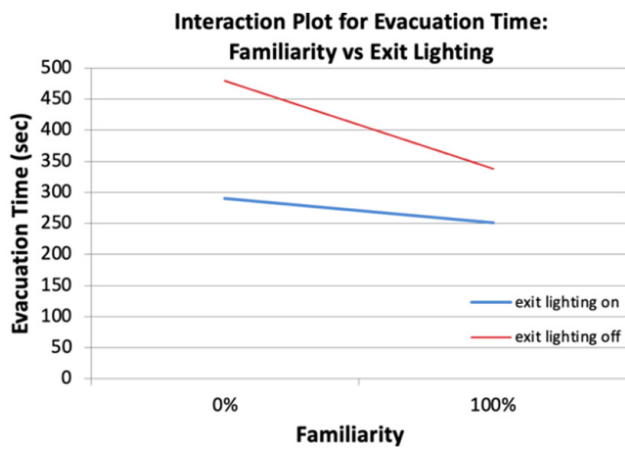

(b)

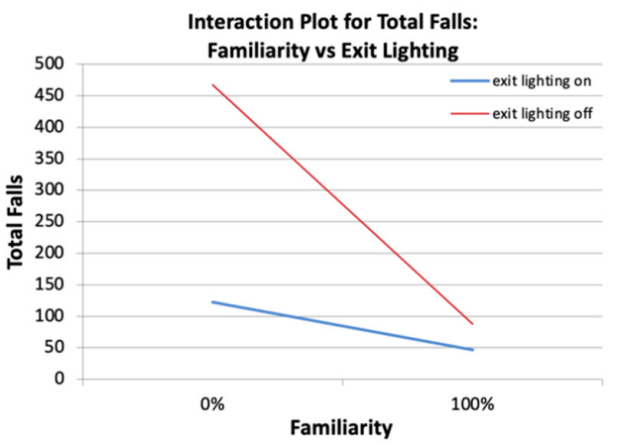

(d)

Figure 5. Interaction plots for the effects of emergency exit lighting, crowd density, and familiarity on (a,b) evacuation time; and $(\mathbf{c}, \mathbf{d})$ total number of falls. 
Next, a $2 \times 2$ independent ANOVA was performed on evacuation time with environmental familiarity $(0 \%, 100 \%)$ and emergency lighting (on, off) as between factors. The main and interaction effects were significant (see Table 4). A $3 \times 2$ independent ANOVA was performed on total number of falls, with crowd density (low, medium, and high) and emergency lighting (on, off) as between factors. The main and interaction effects were significant (see Table 4). Post hoc tests with Tukey HSD corrections show that all levels of crowd density differed significantly between conditions: low-medium, $p=0.001$; medium-high, $p<0.001$; low-high, $p<0.001$. A $2 \times 2$ independent ANOVA was performed on total number of falls, with environmental familiarity $(0 \%, 100 \%)$ and emergency lighting (on, off) as between factors. The main and interaction effects were significant (see Table 4).

These statistically significant results in Table 4 show that the emergency lighting led to a greater decrease in evacuation time for crowds unfamiliar with the environment than for crowds with environmental familiarity (see Figure 5). This is logical, because agents familiar with the environment would have already had more knowledge of where the nearest exits were. The total number of falls increased when crowd density increased, for both lighting conditions, but significantly less steeply when emergency lighting was on, and then especially for high crowd density (see Figure 5). The total number of falls followed the same pattern as evacuation time for environmental familiarity. When agents were not familiar with the environment, the emergency lighting significantly decreased the total number of falls. When agents had environmental familiarity, that itself decreased the total number of falls, leaving no room for an additional effect of emergency lighting.

To conclude, emergency lighting seemed only to decrease evacuation times in medium to high crowd densities and for crowds unfamiliar with the environment. Emergency lighting also seemed to decrease the total number of falls, mainly for high crowd density and for a crowd unfamiliar with the environment.

\subsection{Simulation Experiment 2: Effectiveness of Staff at Exits}

A $3 \times 4$ independent ANOVA was performed on evacuation time, with crowd density (low, medium, and high) and staff members per exit $(0,1,2,3)$ as between factors. The main and interaction effects were significant (see Table 5). Post hoc tests with Tukey HSD corrections show that, between conditions, the high level of crowd density differed significantly from low and medium: low-medium, $p=0.690$; medium-high, $p<0.001$; low-high, $p<0.001$. For staff members, between conditions, only 0 versus 2 or 3 staff members per exit differed significantly: $0-1, p=0.190 ; 0-2, p=0.009 ; 0-3, p=0.004 ; 1-2$, $p=0.651 ; 1-3, p=0.509 ; 2-3, p=0.996$.

These statistically significant results in Table 5 indicate that the presence of staff members at exits seemed to decrease the evacuation time (given the assumptions made), but only for the medium and high crowd density conditions. For low crowd density, the influence of staff members was not statistically significant (see Figure 6). It is important to note, however, that staff members were located close to exits, so the results could have differed if they had been guiding people all around the room.

Next, a $2 \times 4$ independent ANOVA was performed on evacuation time, with environmental familiarity $(0 \%, 100 \%)$ and staff members per exit $(0,1,2,3)$ as between factors. The main and interaction effects were significant (see Table 5). Post hoc tests with Tukey HSD corrections show that, between conditions, only 0 versus 2 or 3 staff members per exit differed significantly: $0-1, p=0.170 ; 0-2, p=0.007 ; 0-3, p=0.003 ; 1-2, p=0.631 ; 1-3$, $p=0.486 ; 2-3, p=0.996$.

A $3 \times 4$ independent ANOVA was performed on total number of falls, with crowd density (low, medium, and high) and staff members per exit $(1,2,3,4)$ as between factors. The main and interaction effects were significant (see Table 5). Post hoc tests with Tukey HSD corrections show all levels of crowd density differed significantly between conditions: low-medium, $p<0.001$; medium-high, $p<0.001$; low-high, $p<0.001$. For staff members, between conditions, only 0 versus 1,2 , or 3 staff members per exit differed significantly: $0-1, p<0.001 ; 0-2, p<0.001 ; 0-3, p<0.001 ; 1-2, p=0.255 ; 1-3, p=0.112 ; 2-3, p=0.977$. 
Table 5. Results of simulation experiment 2: effectiveness of staff at exits.

\begin{tabular}{cccc}
\hline Factor & $d f$ & $F$ & $p$ \\
\hline Effects of crowd density and staff members on evacuation time, $3 \times 4$ ANOVA & \\
\hline Crowd density & 2 & 79.28 & 0.000 \\
Staff members & 3 & 4.79 & 0.003 \\
Crowd density $\times$ Staff members & 6 & 5.29 & 0.000 \\
\hline Effects of environmental familiarity and staff members on evacuation time, $2 \times 4$ ANOVA \\
\hline Environmental familiarity & 1 & 244.21 & 0.000 \\
Staff members & 3 & 5.05 & 0.002 \\
Environmental familiarity $\times$ Staff members & 3 & 9.68 & 0.000 \\
\hline Effect of crowd density and staff members on total number of falls, $3 \times 4$ ANOVA & \\
\hline Crowd density & 2 & 926.51 & 0.000 \\
Staff members & 3 & 23.75 & 0.000 \\
Crowd density $\times$ Staff members & 6 & 11.32 & 0.000 \\
\hline Effect of environmental familiarity and staff members on total number of falls, $2 \times 4$ ANOVA \\
\hline Environmental familiarity \\
Staff members & 1 & 284.36 & 0.000 \\
Environmental familiarity $\times$ Staff members & 3 & 12.51 & 0.000 \\
\hline
\end{tabular}

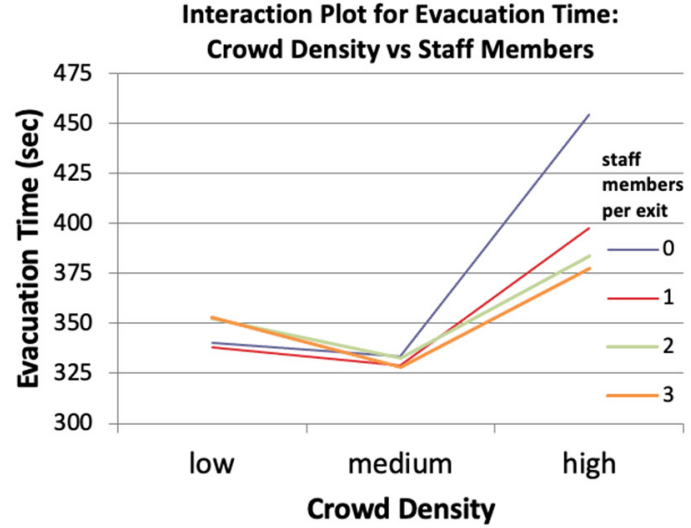

(a)

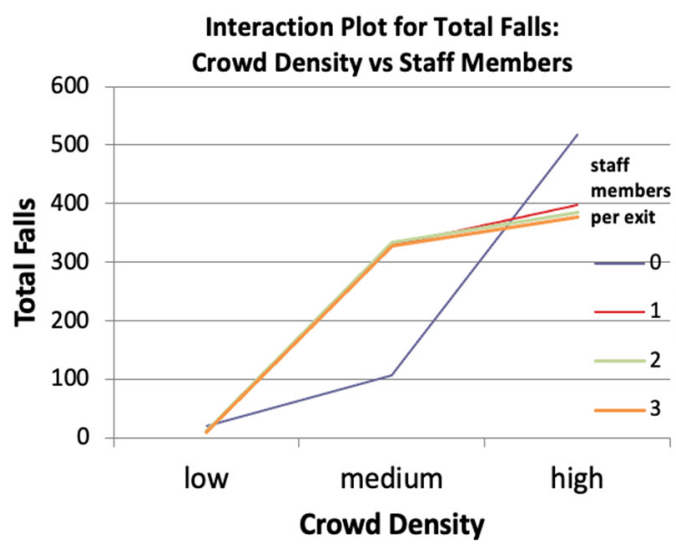

(c)

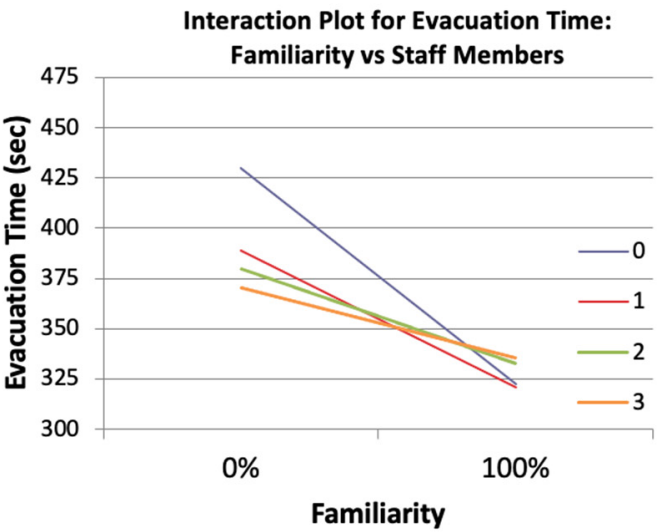

(b)

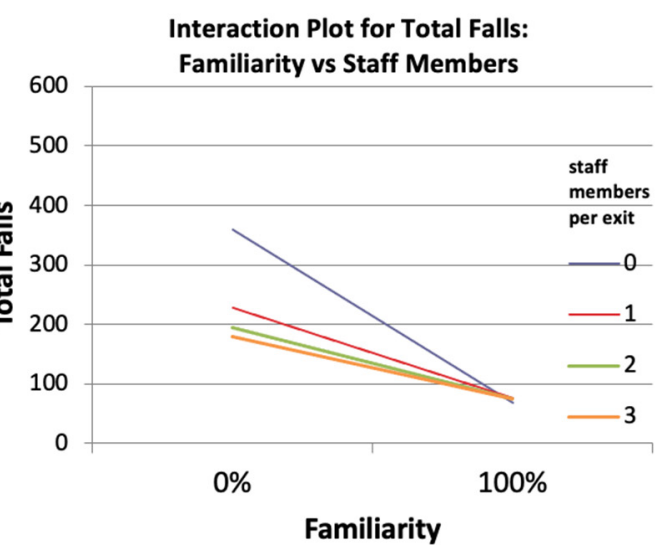

(d)

Figure 6. Interaction plots for the effects of staff member agents at exits, crowd density, and familiarity on (a,b) evacuation time; and (c,d) total number of falls. 
A $2 \times 4$ independent ANOVA was performed on total number of falls, with environmental familiarity $(0 \%, 100 \%)$ and staff members per exit $(0,1,2,3)$ as between factors. The main and interaction effects were significant (see Table 5). Post hoc tests with Tukey HSD corrections show that, between conditions, only 1 versus 3 and 2 versus 3 staff members per exit did not differ significantly: $0-1, p=0.001 ; 0-2, p<0.001 ; 0-3, p<0.001 ; 1-2, p<0.01$; $1-3, p=0.361 ; 2-3, p=0.991$.

These statistically significant results in Table 5 show that, when looking at the interaction effect of staff member agents and environmental familiarity on number of falls, the presence of staff member agents was only beneficial to passenger agents who were unfamiliar with the environment. This is because agents familiar with the environment would have already moved to the nearest exit and naturally passed the staff member agents. The presence of staff member agents seemed to decrease the total number of falls, but this effect diminished beyond the presence of one staff member agent (see Figure 6). When looking at the interaction effect of staff member agents and environmental familiarity, it again seemed that staff member agents were only decreasing the total number of falls when all passenger agents were unfamiliar with the environment. Again, there did not seem to be a difference in the increase in total number of falls between one, two, or three staff member agents. When all passenger agents were familiar with the environment, the presence of staff member agents had no effect.

\subsection{Simulation Experiment 3: Effectiveness of Public Announcement}

A $3 \times 4$ independent ANOVA was performed on evacuation time, with crowd density (low, medium, and high) and cultural cluster distribution $(1,2,3,4)$ as between factors. The main and interaction effects were significant (see Table 6). Post hoc tests with Tukey HSD corrections show all levels of crowd density differed significantly between conditions: low-medium, $p<0.001$; medium-high, $p<0.001$; low-high, $p<0.001$. For cultural clusters, between conditions, all differed significantly except clusters 1 versus 3 and 3 versus $4: 1-2$, $p<0.001 ; 1-3, p=0.273 ; 1-4, p<0.001 ; 2-3, p<0.001 ; 2-4, p<0.001 ; 3-4, p=0.186$.

Table 6. Results of simulation experiment 3: effectiveness of public announcement.

\begin{tabular}{rccc}
\hline Factor & $d f$ & $F$ & $p$ \\
\hline Effects of crowd density and cultural cluster distribuion on evacuation time, & 3 & 4 ANOVA & \\
\hline Crowd density & 2 & 174.14 & 0.000 \\
Cultural cluster distribution & 3 & 28.91 & 0.000 \\
Crowd density $\times$ cultural cluster distribution & 6 & 11.36 & 0.000 \\
\hline Effects of crowd density and cultural cluster distribution on total & number of falls, 3 $\times$ 4 ANOVA \\
\hline Crowd density & 2 & 17019.09 & 0.000 \\
Cultural cluster distribution & 3 & 221.74 & 0.000 \\
Crowd density $\times$ cultural cluster distribution & 6 & 93.34 & 0.000 \\
\hline
\end{tabular}

A $3 \times 4$ independent ANOVA was performed on total number of falls, with crowd density (low, medium, and high) and cultural cluster distribution $(1,2,3,4)$ as between factors. The main and interaction effects were significant (see Table 6). Post hoc tests with Tukey HSD corrections show all levels of crowd density differed significantly between conditions: low-medium, $p<0.001$; medium-high, $p<0.001$; low-high, $p<0.001$. All cultural clusters also differed significantly between conditions: $1-2, p<0.001 ; 1-3, p<0.001$; $1-4, p<0.001 ; 2-3, p<0.001 ; 2-4, p<0.001 ; 3-4, p<0.001$.

The statistically significant simulation results show that cluster 2 (Eastern Europe) and 4 (even mix) had the fastest evacuation times for the majority of situations (see Figure 7). Although this is unexpected, even with a positive effect of public announcement and social influence, there is a third aspect that plays a negative role in the dynamics of the evacuation. The negative impact can be explained by an emergent phenomenon named the faster-isslower effect [71]. This proposes that in evacuations, moving at higher speed (e.g., trying to push your way through a crowded doorway) can sometimes actually increase evacuation 
time given local conflicts. In our simulations, it resulted in more variety in arrival times at the exits, which reduced the evacuation time in certain scenarios. In cluster 2 and especially cluster 4, agents showed a staggered response given the differences in agent's understanding of the announcement, first the agents that understood English, and then the agents influenced by other agents' behaviors, leading to a phased exit and faster evacuation. In clusters 1 (Anglo) and 3 (50-50), however, most of the passenger agents spoke English, and therefore had more chance of understanding the public announcements immediately and therefore taking the nearest exit sooner and together. This created crowd congestion (bottlenecks) at the exit doors and led to slower evacuation. However, this was not the only factor influencing evacuation time, as cluster 4 was not always the fastest to evacuate and cluster 2 was not always the second fastest. In summary, English proficiency and social influence interacted with the faster-is-slower effect to either decrease or increase evacuation time, as detailed above. On top of these effects, the flow capacity of the doors influenced the final evacuation time when the faster-is-slower effect was present.

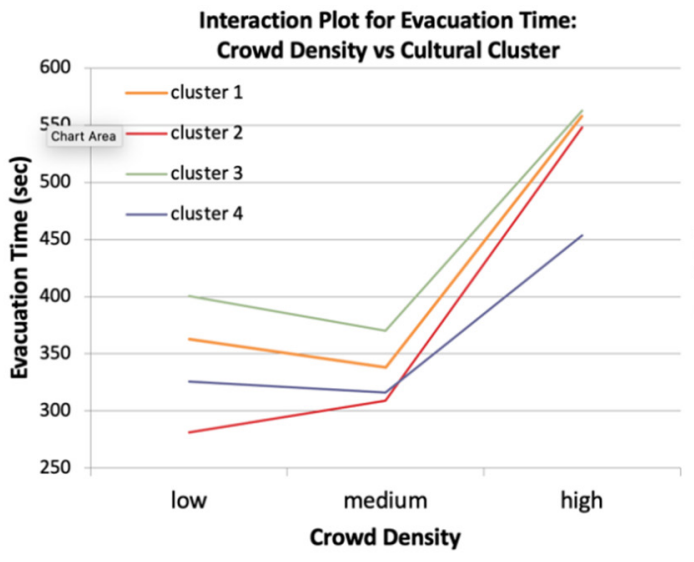

(a)

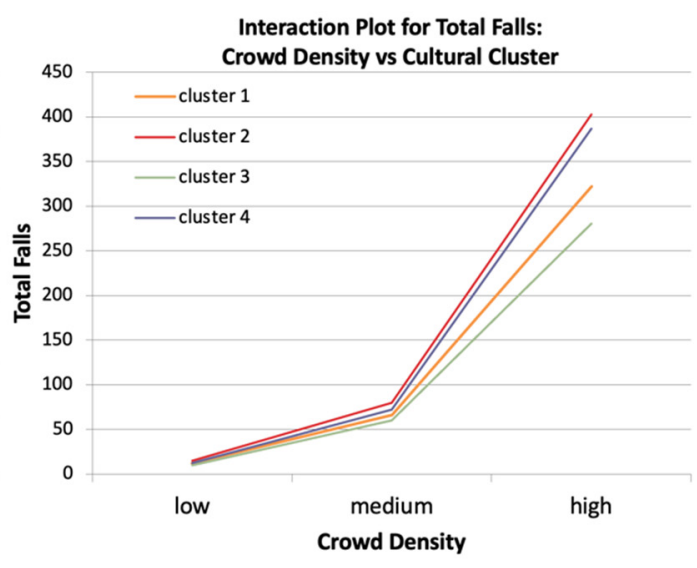

(b)

Figure 7. Interaction plots for the effects of crowd density and cultural clusters on (a) evacuation time; and (b) total number of falls.

Evacuation times were generally lower for medium crowd density than for low or high densities. This could indicate that while social influence was beneficial for evacuation time, as shown in the medium crowd density condition, this effect was canceled out by the congestion in high crowd densities. The opposite was observed for the total number of falls (see Figure 7), however. Total number of falls was lower for clusters 1 (Anglo) and 3 (50-50) than for cluster 2 (Eastern Europe) and cluster 4 (even mix). This effect can be explained by the crowd congestion that happened for clusters 1 and 3 reducing passenger agents' movement speed, and therefore creating fewer falls than in clusters 2 and 4 , where the movement speed was higher due to the lower congestion of the phased evacuation.

\section{Discussion}

\subsection{Discussion of Results}

The findings of the first experiment show that dynamic emergency exit floor lighting can decrease evacuation time, but only for medium and high crowd densities. These findings are consistent with other research showing that environmental familiarity only affects evacuation time in high-density crowds [33]. For high-density crowds, the doors become bottlenecks as passengers unfamiliar with the environment must wait longer to exit as they mostly use the main door. This is consistent with real world observations regarding people's tendencies to exit through the door they entered through, even in emergency situations [72]. However, those who know the environment use a larger number of exits and often therefore avoid bottlenecks. This is contrary to research concerning place scripts (i.e., ingrained cognitive schema for movement and behavior in familiar locations), which 
suggests that such scripts persist even in emergencies, with people still following their usual habitual route through an environment and exiting the way they entered [73]. Accordingly, an important recommendation would be to make the main entrance door very wide to enable rapid exit, and possibly also strategically place columns or pillars to separate flows, reduce crowd pressure, and speed egress (see [74]).

The second emergency communication that was tested was the presence of staff at exits. In the evacuation scenario, the presence of staff at exits decreased the evacuation time only in the high crowd density condition and for crowds unfamiliar with the environment. Additionally, one or two staff members per exit created significant reductions in evacuation time and were sufficient in this scenario. Having more than two staff members did not significantly reduce the evacuation time any further, though. However, the number of falls did not decrease when all passengers were familiar with the environment. Accordingly, it appears that staff members' presence in front of the doors is only beneficial for high-density crowds unfamiliar with the environment. This result is consistent with the emergency lighting results and the results in van der Wal et al. [33], showing familiarity only affects evacuation time in high-density crowds.

Furthermore, it seems that the location of staff has an important effect on evacuation time, as investigated by Formolo et al. [75]. Their results showed a more consistent influence of staff in guiding people to the nearest exit, stimulating a faster evacuation. Furthermore, their research found that the quantity, location, and professionalism of staff are important factors for a successful evacuation. The outcome of this work similarly reflects the positive impact of increasing the number of staff members. A cost-effective recommendation, therefore, is only to use emergency lighting and staff members at exits of very busy terminals, as they have little effect on medium to low crowd densities. One explanation for the effectiveness of floor lighting in high crowd densities would be that the crowd blocks people's line of vision (e.g., a very tall person in front), so they cannot see the usual signs and exits that people in lower density crowds can. This should be interpreted with caution, however, as the simulations were based on a 'neutral' scenario, without environmental features such as walls, pillars, and gradients. In a more complex environment, results may differ.

The third emergency communication strategy that was tested was the English spoken public announcement that requests people to stay calm and evacuate through the nearest exit. The results of simulations evaluating the effectiveness of this public announcement showed that those clusters where fewest people speak English (clusters 2 and 4) have, in general, the fastest evacuation times for the tested scenarios. When looking at the number of falls, the opposite is true in that a lower total number of falls is observed for clusters 1 and 3 , where more than half speak English. Both effects were unexpected, as at an individual level, understanding public announcements should lead to quicker evacuation. However, at a group level it shows different emergent patterns, likely due to the faster-is-slower effect [71], where the distribution of different nationalities (and therefore different English comprehension levels) created a phased evacuation process, in which passengers evacuated one group after another, thereby preventing bottlenecks. Indeed, the clusters with most English speakers were the slowest due to the bottlenecks created by the simultaneous exit. This implies that multicultural crowds could reduce evacuation time in buildings with restricted exit room where bottlenecks may occur.

Although this faster-is-slower effect may occur in simulations, it is also important in real life to consider the delay between those who evacuated first (due to understanding the announcement) and those who evacuated second (by following the first group even though they did not understand the announcement). While a small delay may reduce crowding and therefore reduce overall evacuation time, too long a delay between these two groups could well increase overall evacuation time-in other words, a phased exit could easily become a slow exit if the second group does not realize what the first group is doing. Finally, we should note that if an agent does not understand the public announcement, it can still decide to evacuate. In such cases, the agent keeps walking randomly, until it either 
receives enough social influence (perception of fear beliefs from other agents), or sees the fire itself, to decide to evacuate.

\subsection{Strengths and Limitations}

The strength of this work is its novelty that arose from the inclusion of socio-cultural aspects in emergency communication and its examination by using agent-based simulation of emergencies with multi-cultural crowds. The simulations can estimate the effectiveness of new emergency communication solutions and inform policymakers and emergency managers about risky situations and possible solutions, assuming that it sufficiently represents reality and is credible. We propose this work can be subjected to validation tests via virtual reality experiments (e.g., [21]), where the effects of different emergency communications can be examined on participants from different cultures. The characteristics of the evacuating crowd, building, and emergency communications can be varied more easily than in the real world.

There are several limitations of this work, however. First, the agent-based model that was used for the implementation of the emergency communications [33] only includes a sub-set of the socio-cultural, cognitive, and emotional parameters identified during the development of the model. Conversely, as the number of modeled parameters increases, the analysis of the results becomes more complex and challenging. Second, the results of the simulations should only be used as an indication. We have attempted to make this indication as credible as possible (by basing assumptions on background literature, empirical research, and stakeholders interactions); however all models are significant simplifications of real-world conditions. Third, these outcomes cannot be directly generalized to complex environments, where the environmental characteristics (such as stairways and hallways) will interact with the human behaviors under examination. This work limits itself to making projections about the influence of emergency communication and human behavior on the evacuation process. All the emergency communications were tested in an empty room with four exits. In real life, however, these effects would be combined with the influences of the environment itself, such as corridors, number of exits, stairs, and obstacles. This research could therefore be extended by investigating the combined effect of these elements with the environment (e.g., [76]), but would require the model to be suitably updated to capture these interactions. Finally, although we have modeled evacuation following a fire, we have not included specific hazards related to fires in our model, such as smoke and heat and their effects on human perception and health (e.g., [77]). We chose not to do so here to manage the complexity and interpretability of our simulations, and also because our primary focus here was on understanding human evacuation behavior. So, the fire in our model is currently a generic example of an incident that may necessitate evacuation (e.g., fire, terrorism, accidents), but future research could explore such specific hazards in more detail.

\subsection{Implications for Theory and Practice}

There are a number of implications arising from this work. First, we hope this research shows the potential for testing emergency communications with computer simulations before examining their performance in real-world settings. Simulation allows for greater opportunities, including more repetitions, more explorations, a broader scope of experimental conditions, fewer costs, and fewer ethical concerns. It can help in both preparing for different emergency scenarios as well as predicting the effectiveness of emergency communications under different conditions. Second, transport operators and crowd management professionals could use agent-based models to test in silico competing approaches for crowd management, thereby choosing the best solution proactively. Third, simulations could also support periodic safety and security risk assessments, resulting from, for example, changes in the environment, procedures, new communication measures and/or technologies [78]. Fourth, policymakers could also use these models to help identify new regulations and standards for emergency communication [79]. 


\section{Conclusions}

Evacuation preparedness is essential to reduce common risky behaviors, ensure faster evacuations, and increase the probability of survival. Here, we demonstrated that agentbased modeling can be used to explore various hypotheses regarding evacuee response, testing competing theories, and identifying strategies for further analyses (e.g., real-world experiments and implementation). This research focused on socio-cultural differences in multicultural crowds that are currently unaddressed in evacuation preparations [7]. Three emergency communication strategies were implemented within an existing ABM evacuation model named IMPACT: (1) dynamic emergency exit floor lighting guiding people to exits, (2) staff at exits giving verbal and physical instructions to evacuate, and (3) an English spoken public announcement to evacuate using the nearest exit. Simulation results indicate that (1) dynamic emergency exit floor lighting can decrease evacuation time, but only for medium and high crowd densities; (2) the presence of staff at exits decreased the evacuation time only in the high crowd density condition and for crowds unfamiliar with the environment, whereby one or two staff members per exit created significant reductions in evacuation time and were sufficient in this scenario; and (3) cultures where the fewest people speak English had the fastest evacuation times for the English spoken announcement, which was unexpected but can be explained by this creating a more phased evacuation to avoid congestion in environments constrained by capacity. The strength of this work is its inclusion of socio-cultural aspects in emergency communication and its examination by using agent-based simulations. The limitations concern generalizing these outcome results to more complex environments, where the environmental characteristics, such as stairways and hallways, will interact with the human behaviors under examination. In conclusion, this work shows the potential of testing emergency communications with computer simulations before examining their performance in real-world settings, and helping safety practitioners prepare for different emergency scenarios.

Author Contributions: Conceptualization, C.N.v.d.W., D.F. and M.A.R.; methodology, C.N.v.d.W.; software, D.F. and C.N.v.d.W.; validation, C.N.v.d.W. and D.F.; writing-original draft preparation, C.N.v.d.W.; writing-review and editing, C.N.v.d.W., D.F., M.A.R. and S.G.; funding acquisition, C.N.v.d.W. All authors have read and agreed to the published version of the manuscript.

Funding: This research was funded by European Union's Horizon 2020 research and innovation programme: Marie-Sklodowska-Curie, grant agreement 748647.

Institutional Review Board Statement: Not applicable.

Informed Consent Statement: Not applicable.

Data Availability Statement: Raw data available: https:/ / doi.org/10.6084/m9.figshare.14410388.v1.

Conflicts of Interest: The authors declare no conflict of interest. The funders had no role in the design of the study; in the collection, analyses, or interpretation of data; in the writing of the manuscript, or in the decision to publish the results.

\section{Appendix A}

Table A1. Factors and levels in the simulation experiments.

\begin{tabular}{|c|c|c|}
\hline Parameter & Setting & Meaning \\
\hline Environmental Familiarity & $50 \%$ & $\begin{array}{l}\text { When not specified in the experiment, it is assumed that } 50 \% \text { of the people are } \\
\text { familiar with the environment and will take the nearest exit, while the remaining } \\
50 \% \text { will take the main exit. }\end{array}$ \\
\hline Help & Off & People do not help other people that fall. \\
\hline Falls & On & People are able to fall. \\
\hline Contagion model & On & There is social influence of fear and beliefs. \\
\hline \%children & 15 & $\begin{array}{c}15 \% \text { of the passengers are children, because we chose a ratio of } 70 / 15 / 15 \text { for } \\
\text { adults/children/elderly, respectively. }\end{array}$ \\
\hline \%elderly & 15 & $15 \%$ of the passengers are elderly. \\
\hline \%adults & 70 & $\begin{array}{l}70 \% \text { of the passengers are adults (this is not set directly, but is calculated as } 100 \% \\
\text { with the } \% \text { of children and elderly subtracted). }\end{array}$ \\
\hline
\end{tabular}


Table A1. Cont.

\begin{tabular}{|c|c|c|}
\hline Parameter & Setting & Meaning \\
\hline \%people travelling alone & 50 & $50 \%$ of the people are travelling alone, the remaining $50 \%$ in groups. \\
\hline Group ratios & $50-30-20$ & We assume more people travel in groups of $2(50 \%)$ than $3(30 \%)$ or $4(20 \%)$. \\
\hline \%females & 50 & $50 \%$ are females, $50 \%$ are males. \\
\hline Cultural Clusters & 11 & Represent the 11 clusters from Ronen and Shenkar [59]. \\
\hline Crowd congestion threshold & 5 & $\begin{array}{l}\text { Crowd congestion starts from } 5 \text { people } / \mathrm{m}^{2} \text {, where people cannot maintain their } \\
\text { own pace of movement anymore. }\end{array}$ \\
\hline Public announcement & 60 & Every $60 \mathrm{~s}$, there is one announcement. \\
\hline Fire alarm & 60 & Every $60 \mathrm{~s}$, there is one alarm. \\
\hline Start first time fire alarm & 180 & $180 \mathrm{~s}$ after the fire started, the alarm starts. \\
\hline Start first time public announcement & 20 & $20 \mathrm{~s}$ after the fire alarm, the public announcement starts. \\
\hline Fire present & Always & At the first second. \\
\hline Fire location & Random & $\begin{array}{l}\text { Randomly chosen, but always a minimum of } 3 \mathrm{~m} \text { away from an exit for a fair } \\
\text { comparison of simulations. }\end{array}$ \\
\hline $\begin{array}{l}\text { Cultural cluster distribution } \\
\text { Fire radius }\end{array}$ & $\begin{array}{c}9.09 \\
3\end{array}$ & $\begin{array}{l}\text { People evenly divided over all } 11 \text { clusters: } 9.09 \% \text { per cluster. } \\
3 \mathrm{~m} \text {. }\end{array}$ \\
\hline Communication distance & 5 & $\begin{array}{l}5 \mathrm{~m} \text {, because public distance is } 12-25 \text { feet }(3.7-7.6 \mathrm{~m}) \text {, assuming voice as the main } \\
\text { modality of communication among people (see }[80,81]) \text {. }\end{array}$ \\
\hline Protocol distance & 2 & $2 \mathrm{~m}$ : staff walk randomly around the exit to give instructions, within $2 \mathrm{~m}$ from the \\
\hline $\begin{array}{l}\text { Initial value fear, belief, desire, } \\
\text { intention. }\end{array}$ & 0 & \\
\hline Initial position agent & Random & Randomly chosen from all patches in the environment. \\
\hline Initial heading agent & Random & Randomly chosen from 360 degrees. \\
\hline $\begin{array}{l}\text { Maximum amount of people per } \\
\text { square meter }\end{array}$ & 8 & Based on Still [82]. \\
\hline Congestion speed factor & Speed $\times$ factor & $\begin{array}{l}\text { When there is congestion, agents slow down their speed as follows: [speed } \times 0.625 \\
(8 \text { people); speed } \times 0.75 \text { (7 people); speed } \times 0.875(6 \text { people); speed } \times 0.95 \\
(5 \text { people) }]\end{array}$ \\
\hline Helping behavior & Rule & $\begin{array}{l}\text { People can only help } 1 \text { person at the same time. When a person helps, they are } \\
\text { 'waiting' next to the fallen person until this person stands up, then they continue } \\
\text { moving too. }\end{array}$ \\
\hline Group membership and behavior & Rule & $\begin{array}{l}\text { There is always one leader, NOT a child, that decides where the group will move to. } \\
\text { The whole group moves together on the same square meter. Only the leader will } \\
\text { decide to help or not. The rest will 'wait' with him/her while helping. If the leader } \\
\text { dies, then the group 'splits up', so the others do not 'wait' for the leader to continue, } \\
\text { but continue by themselves. }\end{array}$ \\
\hline $\begin{array}{l}\text { Group formations } \\
\text { Length of fall }\end{array}$ & $\begin{array}{l}\text { Evenly divided } \\
\qquad 30\end{array}$ & $\begin{array}{l}\text { Children, elderly, females/males are evenly divided between all groups. } \\
30 \mathrm{~s} .\end{array}$ \\
\hline Egress flowrate at each exit & 5.4667 & $\begin{array}{c}\text { The maximum is } 6 \text { people per meter per second, based on the egress flowrate of } \\
82 \text { people per minute per meter, which is } 1.3667 \text { people } / \text { meter } / \text { second } \times 4 \text { (doors } \\
\text { are } 4 \mathrm{~m} \text { wide) }=5.4667 \text { people per exit door per second [82]. }\end{array}$ \\
\hline
\end{tabular}

Table A2. General parameter settings for all simulation experiments.

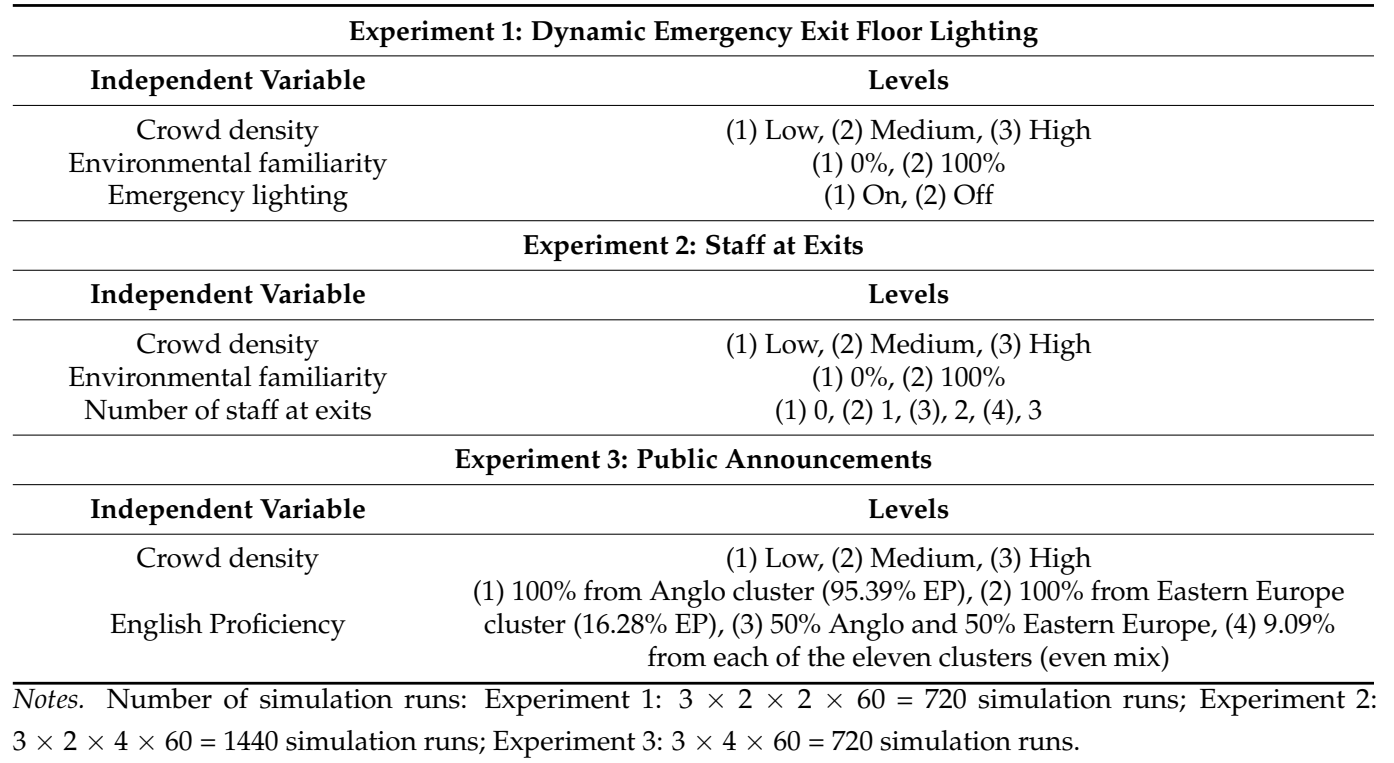




\section{References}

1. Health and Safety Executive. Managing Crowds Safely. A Guide for Organisers at Events and Venues. 2000. Available online: https:/ / www.hse.gov.uk/ (accessed on 1 August 2017).

2. London Resilience Partnership. Communicating with the Public Framework. June 2014. Available online: https:/ /www.london. gov.uk/sites/default/files/london_resilience_partnership_strategy_2020-2023_v3.4.pdf (accessed on 1 August 2017).

3. National Retail Federation. Effective Crowd Management. Guidelines on Maintaining the Safety and Security of Your Customers, Employees and Store. 2014. Available online: http:/ /NRF.com/crowdmanagement (accessed on 1 August 2017).

4. Reynolds, B.; Seeger, M.W. Crisis and emergency risk communication as an integrative model. J. Health Commun. 2005, 10, 43-55. [CrossRef]

5. Vries, P.W.; Galetzka, M.; Gutteling, J.M. Inzet Communicatie bij Crowd Management en Crowd Control; Universiteit Twente-Faculteit Gedragswetenschappen: Enschede, The Netherlands, 2013.

6. WorkSafe Victoria. Crowd Control at Venues and Events: A Practical Occupational Health and Safety Guide. 2007. Available online: https:/ / www.worksafe.vic.gov.au/resources/crowd-control-venues-and-events-practical-occupational-health-andsafety-guide (accessed on 1 August 2017).

7. Santos, G.; Aguirre, B.E. A Critical Review of Emergency Evacuation Simulation Models; Disaster Research Center: Newark, DE, USA, 2004. Available online: http:/ / udspace.udel.edu/handle/19716/299 (accessed on 1 August 2017).

8. Fothergill, A.; Maestes, E.G.; Darlington, J.D. Race, ethnicity and disasters in the United States: A review of the literature. Disasters 1999, 23, 156-173. [CrossRef]

9. Hoffman, S. Preparing for disaster: Protecting the most vulnerable in emergencies. Univ. Calif. Davis Law Rev. 2008, $42,1491-1547$.

10. Howard, A.; Agllias, K.; Bevis, M.; Blakemore, T. "They'll tell us when to evacuate": The experiences and expectations of disaster-related communication in vulnerable groups. Int. J. Disaster Risk Reduct. 2017, 22, 139-146. [CrossRef]

11. Rowan, K.E. Why rules for risk communication are not enough: A problem-solving approach to risk communication. Risk Anal. 1994, 14, 365-374. [CrossRef]

12. Turner, D.; Evans, W.; Kumlachew, M.; Wolshon, B.; Dixit, V.; Sisiopiku, V.; Islam, S.; Anderson, M. Issues, practices, and needs for communicating evacuation information to vulnerable populations. Transp. Res. Rec. J. Transp. Res. Board 2010, 2196, 159-167. [CrossRef]

13. Dombroski, M.; Fischhoff, B.; Fischbeck, P. Predicting emergency evacuation and sheltering behavior: A structured analytical approach. Risk Anal. 2006, 26, 1675-1688. [CrossRef] [PubMed]

14. Kim, J.; Oh, S.S. Confidence, knowledge, and compliance with emergency evacuation. J. Risk Res. 2015, 18, 111-126. [CrossRef]

15. Knuth, D.; Kehl, D.; Hulse, L.; Spangenberg, L.; Brahler, E.; Schmidt, S. Risk perception and emergency experience: Comparing a representative German sample with German emergency survivors. J. Risk Res. 2015, 18, 581-601. [CrossRef]

16. Mallett, L.; Vaught, C.; Brnich, M.J., Jr. Sociotechnical communication in an underground mine fire: A study of warning messages during an emergency evacuation. Saf. Sci. 1993, 16, 709-728. [CrossRef]

17. Riad, J.K.; Norris, F.H.; Ruback, R.B. Predicting evacuation in two major disasters: Risk perception, social influence, and access to resources. J. Appl. Soc. Psychol. 1999, 29, 918-934. [CrossRef]

18. Dekel, S.; Bonanno, G.A. Changes in trauma memory and patterns of posttraumatic stress. Psychol. Trauma Theory Res. Pract. Policy 2013, 5, 26-34. [CrossRef]

19. Roseman, M. Surviving memory: Truth and inaccuracy in Holocaust testimony. J. Holocaust Educ. 1999, 8, 1-20. [CrossRef]

20. Van der Wal, C.N.; Robinson, M.A.; Gwynne, S.; Bruine de Bruin, W. Evacuation behaviors and emergency communications: An analysis of real-world incident videos. Saf. Sci. 2021, 136, 105121. [CrossRef]

21. Drury, J.; Cocking, C.; Reicher, S.; Burton, A.; Schofield, D.; Hardwick, A.; Graham, D.; Langston, P. Cooperation versus competition in a mass emergency evacuation: A new laboratory simulation and a new theoretical model. Behav. Res. Methods 2009, 41, 957-970. [CrossRef]

22. Gwynne, S.M.V.; Kuligowski, E.D.; Boyce, K.E.; Nilsson, D.; Robbins, A.P.; Lovreglio, R.; Thomas, J.R.; Roy-Poirier, A. Enhancing egress drills: Preparation and assessment of evacuee performance. Fire Mater. 2019, 43, 613-631. [CrossRef]

23. Bryson, J.J.; Ando, Y.; Lehmann, H. Agent-based modelling as scientific method: A case-study analyzing primate social behaviour. Philos. Trans. R. Soc. Lond. B Biol. Sci. 2007, 362, 1685-1699. [CrossRef] [PubMed]

24. Wilensky, U.; Rand, W. An Introduction to Agent-Based Modeling: Modeling Natural, Social, and Engineered Complex Systems with NetLogo; MIT Press: Cambridge, MA, USA, 2015.

25. Hughes, H.P.N.; Clegg, C.W.; Robinson, M.A.; Crowder, R.M. Agent-based modelling and simulation: The potential contribution to organizational psychology. J. Occup. Organ. Psychol. 2012, 85, 487-502. [CrossRef]

26. Crowder, R.M.; Robinson, M.A.; Hughes, H.P.N.; Sim, Y.W. The development of an agent-based modeling framework for simulating engineering team work. IEEE Trans. Syst. Man Cybernet. Part A Syst. Hum. 2012, 42, 1425-1439. [CrossRef]

27. Reilly, A.C.; Dillon, R.L.; Guikema, S.D. Agent-Based Models as an Integrating Boundary Object for Interdisciplinary Research. Risk Anal. 2018. [CrossRef]

28. Gorbil, G.; Gelenbe, E. Opportunistic communications for emergency support systems. Procedia Comp. Sci. 2011, 5, 39-47. [CrossRef]

29. Ji, X.; Weng, W.; Fan, W. Cellular automata-based systematic risk analysis approach for emergency response. Risk Anal. 2008, 28, 1247-1260. [CrossRef] [PubMed] 
30. Medeiros, L.; van der Wal, C.N. An Agent-Based Model Predicting Group Emotion and Misbehaviours in Stranded Passengers. In Proceedings of the 18th EPIA Conference on Artificial Intelligence, Porto, Portugal, 5-8 September 2017; Springer: Berlin/Heidelberg, Germany, 2017; pp. 620-627.

31. Haer, T.; Botzen, W.J.; Moel, H.; Aerts, J.C. Integrating household risk mitigation behavior in flood risk analysis: An agent-based model approach. Risk Anal. 2017, 37, 1977-1992. [CrossRef] [PubMed]

32. Van der Wal, C.N.; Guiricin, A.; Turhan, U.; Sengur, F.; Tedeschi, A.; Rozzi, S.; Golfetti, A.; Robinson, M. Requirements for the Multi-Cultural Emergency Communication Framework; European Commission: Brussels, Belgium, 2016.

33. van der Wal, C.N.; Formolo, D.; Robinson, M.; Minkov, M.; Bosse, T. Simulating Crowd Evacuation with Socio-Cultural, Cognitive and Emotional Elements. In Transactions on Computational Collective Intelligence XXVII; Springer: Cham, Switzerland, 2017. [CrossRef]

34. Fridolf, K.; Nilsson, D.; Frantzich, H. Fire evacuation in underground transportation systems: A review of accidents and empirical research. Fire Technol. 2013, 49, 451-475. [CrossRef]

35. Samochine, D.; Boyce, K.; Shields, T. An investigation into staff behaviour in unannounced evacuations of retail storesImplications for training and fire safety engineering. Fire Saf. Sci. 2005, 8, 519-530. [CrossRef]

36. Shields, T.; Boyce, K.A. study of evacuation from large retail stores. Fire Saf. J. 2000, 35, 25-49. [CrossRef]

37. Ibrahim, A.M.; Venkat, I.; Subramanian, K.; Khader, A.T.; Wilde, P.D. Intelligent evacuation management systems: A review. ACM Trans. Intell. Syst. Technol. (TIST) 2016, 7, 36. [CrossRef]

38. Zhang, Q.; Chen, T.; Lv, X.Z. New framework of intelligent evacuation system of buildings. Procedia Eng. 2014, 71, 397-402. [CrossRef]

39. Amirgaliyev, Y.; Yunussov, R.; Mamyrbayev, O. Optimization of people evacuation plans on the basis of wireless sensor networks. Open Eng. 2016, 6, 206-213. [CrossRef]

40. Challenger, R.; Clegg, C.W.; Robinson, M.A. Understanding Crowd Behaviours: Practical Guidance and Lessons Identified; TSO: London, UK, 2010; Volume 1.

41. Tan, M.S.; Said, S.B. Linguistic Landscape and Exclusion: An Examination of Language Representation in Disaster Signage in Japan. In Conflict, Exclusion and Dissent in the Linguistic Landscape; Palgrave Macmillan: London, UK; pp. 145-169.

42. Challenger, R.; Clegg, C.W.; Robinson, M.A. Understanding Crowd Behaviours; TSO: London, UK, $2010 ;$ Volume 2.

43. Luh, P.B.; Wilkie, C.T.; Chang, S.C.; Marsh, K.L.; Olderman, N. Modeling and optimization of building emergency evacuation considering blocking effects on crowd movement. IEEE Trans. Autom. Sci. Eng. 2012, 9, 687-700. [CrossRef]

44. Yang, L.Z.; Zhao, D.L.; Li, J.; Fang, T.Y. Simulation of the kin behavior in building occupant evacuation based on cellular automaton. Build. Environ. 2005, 40,411-415. [CrossRef]

45. Reicher, S.D. 'The Battle of Westminster': Developing the social identity model of crowd behaviour in order to explain the initiation and development of collective conflict. Eur. J. Soc. Psychol. 1996, 26, 115-134. [CrossRef]

46. Von Sivers, I.; Templeton, A.; Koster, G.; Drury, J.; Philippides, A. Humans do not always act selfishly: Social identity and helping in emergency evacuation simulation. Transp. Res. Procedia 2014, 2, 585-593. [CrossRef]

47. Ronchi, E.; Nilsson, D. Fire evacuation in high-rise buildings: A review of human behaviour and modelling research. Fire Sci. Rev. 2013, 2, 7. [CrossRef]

48. Hashim, M.J.; Alkaabi, M.S.K.M.; Bharwani, S. Interpretation of way-finding healthcare symbols by a multicultural population: Navigation signage design for global health. Appl. Ergon. 2014, 45, 503-509. [CrossRef]

49. Vilar, E.; Rebelo, F.; Noriega, P. Indoor human wayfinding performance using vertical and horizontal signage in virtual reality. Hum. Factors Ergon. Manuf. Serv. Ind. 2014, 24, 601-615. [CrossRef]

50. Robinson, M.; Cichomska, K.; Davis, M.; Minkov, M.; Blagoev, V.; van der Wal, N.; Turhan, U.; Karbownik, A.; Giuricin, A.; Rozzi, A.; et al. Review and Critical Analysis of Multi-Cultural Crowd Behaviour in Emergencies; European Commission: Brussels, Belgium, 2015.

51. Hofstede, G. Culture's Consequences: Comparing Values, Behaviors, Institutions, and Organizations across Nations, 2nd ed.; Sage: Thousand Oaks, CA, USA, 2001.

52. Reicher, S.; Stott, C.; Cronin, P.; Adang, O. An integrated approach to crowd psychology and public order policing. Polic. Int. J. Police Strateg. Manag. 2004, 27, 558-572. [CrossRef]

53. Farr, A.C.; Kleinschmidt, T.; Yarlagadda, P.; Mengersen, K. Wayfinding: A simple concept, a complex process. Transp. Rev. 2012, 32, 715-743. [CrossRef]

54. Omer, I.; Goldblatt, R. The implications of inter-visibility between landmarks on wayfinding performance: An investigation using a virtual urban environment. Comput. Environ. Urban Syst. 2007, 31, 520-534. [CrossRef]

55. Palttala, P.; Boano, C.; Lund, R.; Vos, M. Communication gaps in disaster management: Perceptions by experts from governmental and non-governmental organizations. J. Conting. Crisis Manag. 2012, 20, 2-12. [CrossRef]

56. Owen, M.; Galea, E.R.; Lawrence, P.J. The Safeguard Validation Set-Svgds1 a Guide to the Data and Validation Procedures, Fire Safety Engineering Group; University of Greenwich: London, UK, 1996.

57. Galea, E.; Deere, S.; Filippidis, L. The Safeguard Validation Data Set-Sgvds1 a Guide to the Data and Validation Procedures, Fire Safety Engineering Group; University of Greenwich: London, UK, 2012.

58. Kuligowski, E. A Comprehensive Review of 28 Evacuation Models. In Workshop on Building Occupant Movement during Fire Emergencies; Institute of Standards and Technology: Gaithersburg, MD, USA, 2004. 
59. Templeton, A.; Drury, J.; Philippides, A. From mindless masses to small groups: Conceptualizing collective behavior in crowd modeling. Rev. Gen. Psychol. 2015, 19, 215-229. [CrossRef]

60. Zheng, X.; Zhong, T.; Liu, M. Modeling crowd evacuation of a building based on seven methodological approaches. Build. Environ. 2009, 44, 437-445. [CrossRef]

61. Rao, A.S.; Georgeff, M.P. BDI agents: From theory to practice. In Proceedings of the First International Conference on Multiagent Systems, San Francisco, CA, USA, 12-14 June 1995; Volume 95, pp. 312-319.

62. Treur, J. Network-Oriented Modeling; Springer International Publishing: Cham, Switzerland, 2016.

63. Reininger, B.M.; Raja, S.A.; Carrosco, A.S.; Chen, Z.; Adams, B.; McCormick, J.; Rahbar, M.H. Intention to comply with mandatory hurricane evacuation orders among persons living along a coastal area. Disaster Med. Public Health Prep. 2013, 7, 46-54. [CrossRef]

64. Soto, C.J.; John, O.P.; Gosling, S.D.; Potter, J. Age differences in personality traits from 10 to 65: Big five domains and facets in a large cross-sectional sample. J. Pers. Soc. Psychol. 2011, 100, 330-348. [CrossRef]

65. Formolo, D.; van der Wal, C.N. Simulating collective evacuations with social elements. In Conference on Computational Collective Intelligence Technologies and Applications; Springer: Cham, Switzerland, 2020; pp. 160-171.

66. Galea, E.R.; Xie, H.; Lawrence, P.J. Experimental and survey studies on the effectiveness of dynamic signage systems. Fire Saf. Sci. 2014, 11, 1129-1143. [CrossRef]

67. Cosma, G.; Ronchi, E.; Nilsson, D. Way-finding lighting systems for rail tunnel evacuation: A virtual reality experiment with Oculus Rift ${ }^{\circledR}$. J. Transp. Saf. Secur. 2016, 8, 101-117. [CrossRef]

68. Ronen, S.; Shenkar, O. Mapping world cultures: Cluster formation, sources and implications. J. Int. Bus. Stud. 2013, 44, 867-897. [CrossRef]

69. English Proficiency Index. Available online: https:/ /www.ef.co.uk/epi (accessed on 1 August 2017).

70. European Population Distribution. Available online: https:/ / ec.europa.eu/eurostat (accessed on 1 August 2017).

71. Zhou, S.; Chen, D.; Cai, W.; Luo, L.; Low, M.Y.H.; Tian, F.; Tay, V.S.H.; Ong, D.W.S.; Hamilton, B.D. Crowd modeling and simulation technologies. ACM Trans. Model. Comput. Simul. 2010, 20, 1-35. [CrossRef]

72. Gosshandler, W.; Bryner, N.; Madrzykowski, D.; Kuntz, K. Report of the Technical Investigation of the Station Nightclub Fire; US Department of Commerce: Washington, DC, USA, 2005; Volume 1.

73. Donald, I.; Canter, D. Intentionality and fatality during the King's Cross underground fire. Eur. J. Soc. Psychol. 1992, 22, 203-218. [CrossRef]

74. Yanagisawa, D.; Kimura, A.; Tomoeda, A.; Nishi, R.; Suma, Y.; Ohtsuka, K.; Nishinari, K. Introduction of frictional and turning function for pedestrian outflow with an obstacle. Phys. Rev. 2009, 80, 36110. [CrossRef] [PubMed]

75. Formolo, D.; Bosse, T.; van der Wal, C.N. Studying the Impact of Trained Staff on Evacuation Scenarios by Agent-Based Simulation. In Proceedings of the International Conference on Social Informatics, Saint-Petersburg, Russia, 25-28 September 2018; Springer: Cham, Switzerland, 2020; pp. 85-96.

76. Varas, A.; Cornejo, M.D.; Mainemer, D.; Toledo, B.; Rogan, J.; Munoz, V.; Valdivia, J.A. Cellular automaton model for evacuation process with obstacles. Phys. A Stat. Mech. Appl. 2007, 382, 631-642. [CrossRef]

77. Xu, Z.; Lu, X.Z.; Guan, H.; Chen, C.; Ren, A.Z. A virtual reality based fire training simulator with smoke hazard assessment capacity. Adv. Eng. Softw. 2014, 68, 1-8. [CrossRef]

78. Clegg, C.W.; Robinson, M.A.; Davis, M.C.; Bolton, L.; Pieniazek, R.; McKay, A. Applying organizational psychology as a design science: A method for predicting malfunctions in socio-technical systems (PreMiSTS). Des. Sci. 2017, 3, 1-31. [CrossRef]

79. Seeger, M.W. Best practices in crisis communication: An expert panel process. J. Appl. Commun. Res. 2006, 34, 232-244. [CrossRef]

80. Overhill, H. Apple pie proxemics: Edward, T. Hall in the kitchen work triangle. Des. Issues 2014, 30, 67-82. [CrossRef]

81. Proxemics [and Comments and Replies]. Available online: https://www.journals.uchicago.edu/doi/abs/10.1086/200975 (accessed on 1 August 2017).

82. Still, K. Introduction to Crowd Science; CRC Press: Boca Raton, FL, USA, 2004. 